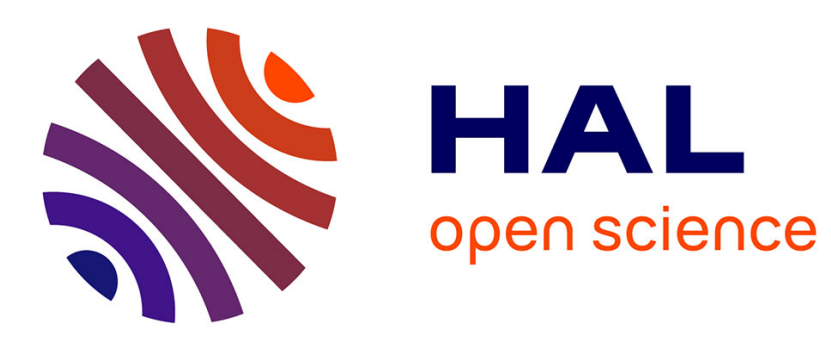

\title{
Cut-free Ordinary Sequent Calculi for Logics Having Generalized Finite-Valued Semantics
}

Arnon Avron, Jonathan Ben-Naim, Beata Konikowska

\section{To cite this version:}

Arnon Avron, Jonathan Ben-Naim, Beata Konikowska. Cut-free Ordinary Sequent Calculi for Logics Having Generalized Finite-Valued Semantics. Logica Universalis, 2007, 1, pp.41-70. 10.1007/s11787006-0003-6 . hal-03466419

\section{HAL Id: hal-03466419 https://hal.science/hal-03466419}

Submitted on 5 Dec 2021

HAL is a multi-disciplinary open access archive for the deposit and dissemination of scientific research documents, whether they are published or not. The documents may come from teaching and research institutions in France or abroad, or from public or private research centers.
L'archive ouverte pluridisciplinaire HAL, est destinée au dépôt et à la diffusion de documents scientifiques de niveau recherche, publiés ou non, émanant des établissements d'enseignement et de recherche français ou étrangers, des laboratoires publics ou privés. 


\title{
Cut-free Ordinary Sequent Calculi for Logics Having Generalized Finite-Valued Semantics
}

\author{
Arnon Avron, Jonathan Ben-Naim and Beata Konikowska
}

\section{Introduction}

For at least seven decades, many-valued logics have been a challenging and fruitful field of research. This research became particularly extensive with the advent of computers, and then information systems, data and knowledge bases, expert systems, and artificial intelligence. In all these fields, many applications have been found for logics having finite-valued semantics.

The applicability of finite-valued semantics has recently been greatly increased by a major generalization of the ordinary many-valued semantics for logical systems: the use of non-deterministic matrices — shortly, Nmatrices $([9,8])$. While preserving all the good properties of the ordinary finite-valued matrices, Nmatrices can be used to provide (generalized) finite-valued semantics for many logics which do not possess semantics based on the former matrices (see $[1,2,3,4,5,6,9]$ ). Two examples of large families of this kind are:

$[8,9]$ : All logics having ordinary sequent calculi with only canonical rules ${ }^{1}$, except those which are exact fragments of classical logic. This family includes, e.g., all logics obtained from classical logic by deleting some rule(s) from its standard sequent calculus.

$[3,4,6]$ : All the paraconsistent LFIs (Logics with Formal Inconsistency) and $\mathrm{C}$-systems considered in $([14,15]$, except for those including the axiom $(l)$, or the axiom $(d)$, or the axiom $(o)$. This family includes thousands of logics. With the growing importance of finite-valued logics, a lot of efforts have been devoted to developing proof systems for such logics (see [10, 16]). For an ordinary $n$-valued semantics, the usual way of doing so in a uniform way is by employing a calculus of $n$-sequents, i.e.: sequents with $n$ components, or "sides" (or an equivalent deduction mechanism based on sets of $n$-signed formulas). Unfortunately,

\footnotetext{
${ }^{1}$ Canonical rules are context-free rules introducing exactly one connective on one of the two sides of the sequent, with the composed formula appearing only once in the conclusion.
} 
such mechanisms, though useful, are much less known than those which are based on ordinary, two sided sequents. Moreover: working with calculi of the latter type has some obvious advantages that make the search for them a worthwhile task:

1. In an ordinary sequent calculus, the answer to the question when a sentence $\varphi$ follows from a finite set of premises $\Gamma$ (which is the central problem of logic) is very simple: $\varphi$ follows from $\Gamma$ iff the sequent $\Gamma \Rightarrow \varphi$ is provable in the system. In other words: the use of two-sided sequents reflects the basic fact that logic is all about consequence relations. In contrast, the use of an $n$-sequents calculus makes even characterization of the consequence relation a rather complicated task, since this can only be done in a roundabout way.

2. The use of two-sided sequents is universal, and independent of any particular semantics. As opposed to this, the use of $n$-sequents relies in an essential way on a specific semantics for a given logic (which might not be the only useful one). We believe that mixing in this way the proof-theory of a logic with its semantics is conceptually wrong. Suppose, for example, that $\varphi$ is valid in $L_{2}$ and that $L_{1}$ is an extension of $L_{2}$. Suppose further that $L_{2}$ has a characteristic $n$-valued matrix, while $L_{1}$ has a k-valued one - say, with $k<n$ (the literature on many-valued logics is full of this type of examples). In this case, finding a proof of $\varphi$ in an $n$-sequent calculus for $L_{2}$ is of no help in finding a proof of $\varphi$ in an $k$-sequent calculus for $L_{1}$ : usually, all the work has to be started from a scratch. On the other hand, when a uniform framework is used (like ordinary two-sided sequent calculi), one can try to develop a calculus for $L_{1}$ by extending a known calculus for $L_{2}$. This is indeed often done successfully ${ }^{2}$ - and in such a case, a proof of $\varphi$ in $L_{2}$ is also a proof of $\varphi$ in $L_{1}$.

3. Since the most important logics (like classical logic, intuitionistic logic and the most famous modal logics) have useful two-sided sequent calculi (or related calculi, like tableaux or resolution ones), the framework of such calculi is well-understood, and a lot of progress has been made (with even more to follow certainly in the future) towards developing their efficient implementations. Clearly, given some logic, it is desirable to be able to adapt such implementations for its use. This may be possible only if a calculus of the same type is available for that logic.

In this paper we show that for a large, central class of (generalized) finitevalued logics, the language of which satisfies a certain minimal expressiveness condition, one can transform a given sound and complete $n$-sequent proof system into an equivalent sound and complete system of ordinary sequents. The expressiveness condition is that we must be able to identify the truth-value of any formula $\varphi$ by determining whether certain formulas uniformly constructed from $\varphi$ are true (i.e.: have designated values) or not (note that we do not need to know the exact truth values of those formulas). Obviously, any language which does not satisfy this condition can (perhaps should) be extended to one which does, so our procedure

\footnotetext{
${ }^{2}$ See [2] for many examples of this sort where $n=4$ and $k=3$.
} 
is quite general. Moreover, our transformation preserves the structures of proofs in the original calculus in the following sense: each rule of the original calculus induces a finite set of rules of the new, two-sided calculus, and a rule is used in a proof resulting from the transformation only if it is induced by a rule used in the original proof. This fact automatically entails a weak form of the cut elimination theorem for the resulting two-sided calculus if the same (weak) theorem obtains for the original one (the weak form says that every sequent which has a proof with cuts has also a cut-free proof).

The present paper is a companion to [7], where we showed special cases of the above transformation for certain important logics, and announced a forthcoming general result and method - which we give in this paper. We also illustrate here that method on several concrete examples of many-valued logics, including a new application for information sources logics.

\section{General Framework for Generalized Finite-valued Logics}

In this section we present the general framework for discussing generalized finitevalued logic which will be used throughout the paper.

\subsection{General Semantics and Consequence Relation}

In what follows $\mathcal{L}$ is a propositional language, $O_{k}(k \geq 0)$ is the set of its $k$-ary connectives, $\mathcal{W}$ is its set of wffs, $p, q, r$ denote propositional variables, $\varphi, \psi, \phi, \tau$ denote arbitrary formulas (of $\mathcal{L}$ ), and $\Gamma, \Delta$ denote finite sets of formulas.

\section{Definition 2.1.}

- $A$ (generalized) finite-valued semantics for $\mathcal{L}$ is a triple $(\mathcal{T}, \mathcal{D}, \mathcal{V})$, where $\mathcal{T}$ is a set of truth values, $\mathcal{D}$, the set of designated values, is a nonempty proper subset of $\mathcal{T}$, and $\mathcal{V}$ is a set of functions from $\mathcal{W}$ to $\mathcal{T}$ (the "admissible", or "legal", valuations). We shall usually use $\mathcal{V}$ also to refer to such a triple, and denote the set of non-designated values by $\mathcal{N}$.

- A formula $\varphi \in \mathcal{W}$ is satisfied by a valuation $v \in \mathcal{V}$, in symbols $v \models \varphi$, if $v(\varphi) \in \mathcal{D}$.

- An (ordinary) sequent $\Sigma=\Gamma \Rightarrow \Delta$ is satisfied by a valuation $v \in \mathcal{V}$, in symbols $v \models \Sigma$, iff either $v$ does not satisfy some formula in $\Gamma$ or $v$ satisfies some formula in $\Delta$.

- A sequent $\Sigma$ is valid if it is satisfied by all valuations $v \in \mathcal{V}$.

- The (Scott) consequence relation on $\mathcal{W}$ defined by $\mathcal{V}$ is the relation $\vdash_{\mathcal{V}}$ on sets of formulas in $\mathcal{W}$ such that, for any $T, S \subseteq W, T \vdash_{\mathcal{V}} S$ iff there exist finite sets $\Gamma \subseteq T, \Delta \subseteq S$ such that the sequent $\Gamma \Rightarrow \Delta$ is valid.

\subsection{Ordinary and Non-deterministic Logical Matrices}

The most common way of defining a set $\mathcal{V}$ of admissible valuations for a language $\mathcal{L}$ is by using the well-known, ordinary logical matrices, defined as follows: 
Definition 2.2. A logical matrix for $\mathcal{L}$ is a triple $\mathcal{M}=(\mathcal{T}, \mathcal{D}, \mathcal{O})$, where $\mathcal{T}$ is a finite, non-empty set of truth values, $\mathcal{D}$ is a non-empty proper subset of $\mathcal{T}$ (containing its designated values), and $\mathcal{O}$ includes a $k$-ary function $\widetilde{\diamond}: \mathcal{T}^{k} \rightarrow \mathcal{T}$ for every $k$-ary connective $\diamond \in O_{k}$.

A valuation in an ordinary matrix $\mathcal{M}$ is defined compositionally, based on the interpretation of the connectives:

Definition 2.3. Let $\mathcal{M}=(\mathcal{T}, \mathcal{D}, \mathcal{O})$ be a logical matrix. A valuation in $\mathcal{M}$ is a function $v: \mathcal{W} \rightarrow \mathcal{T}$ such that

$$
v\left(\diamond\left(\psi_{1}, \ldots, \psi_{k}\right)\right)=\widetilde{\diamond}\left(v\left(\psi_{1}\right), \ldots, v\left(\psi_{k}\right)\right)
$$

for each $k$-ary connective $\diamond \in O_{k}$ and for all $\psi_{1}, \ldots, \psi_{k} \in \mathcal{W}$.

However, many logics which do not possess finite-valued semantics based on finite ordinary matrices can be given generalized finite-valued semantics based on finite non-deterministic matrices, characterized by a set-valued interpretation of connectives:

Definition 2.4. ([8,9]) $A$ non-deterministic matrix (Nmatrix) for $\mathcal{L}$ is a triple $\mathcal{M}=(\mathcal{T}, \mathcal{D}, \mathcal{O})$, where $\mathcal{T}$ is a non-empty set of truth values, $\mathcal{D}$ is a non-empty proper subset of $\mathcal{T}$ (containing its designated values), and $\mathcal{O}$ includes a $k$-ary function $\widetilde{\diamond}: \mathcal{T}^{k} \rightarrow 2^{\mathcal{T}} \backslash\{\emptyset\}$ for every $k$-ary connective $\diamond \in O_{k}$.

Definition 2.5. Let $\mathcal{M}=(\mathcal{T}, \mathcal{D}, \mathcal{O})$ be an Nmatrix. $A$ valuation in $\mathcal{M}$ is a function $v: \mathcal{W} \rightarrow \mathcal{T}$ such that

$$
v\left(\diamond\left(\psi_{1}, \ldots, \psi_{k}\right)\right) \in \widetilde{\diamond}\left(v\left(\psi_{1}\right), \ldots, v\left(\psi_{k}\right)\right)
$$

for each $k$-ary connective $\diamond \in O_{k}$ and for all $\psi_{1}, \ldots, \psi_{k} \in \mathcal{W}$.

As one can see from the above definitions, the value of $v\left(\diamond\left(\psi_{1}, \ldots, \psi_{k}\right)\right)$ is selected out of the whole range of the allowed values in $\widetilde{\diamond}\left(v\left(\psi_{1}\right), \ldots, v\left(\psi_{k}\right)\right)$ separately and independently for each tuple $\left\langle v\left(\psi_{1}\right), \ldots, v\left(\psi_{k}\right)\right\rangle$. Thus, in contrast to ordinary matrices, the valuations provided by Nmatrices are not compositional, and $v\left(\psi_{1}\right), \ldots, v\left(\psi_{k}\right)$ do not uniquely determine $v\left(\diamond\left(\psi_{1}, \ldots, \psi_{k}\right)\right)$.

If for each $\diamond \in O_{k}$ the function $\widetilde{\diamond}$ is singleton-valued, then $\mathcal{M}$ defined as above is equivalent to an ordinary logical matrix. Thus Nmatrices are a generalization of ordinary matrices. More importantly, finite Nmatrices preserve the basic advantages of the finite ordinary, deterministic matrices, like decidability, compactness $([9])$, effectiveness (i.e. the possibility to extend to a full valuation any semivaluation defined on a subset of $\mathcal{W}$ closed under subformulas), and the availability of a uniform method for developing corresponding cut-free $n$-sequent calculi for them. This method is reviewed in the next subsection.

\subsection{Proof Systems Based on $n$-sequents}

Next we recall the basic formalism for reasoning about finite-valued logics, which will be the main tool of our method: $n$-sequents (or their alternate representation 
- sets of signed formulas). In the rest of this section $\mathcal{V}$ is some (generalized) finitevalued semantics. For simplicity, we assume that $\mathcal{T}=\left\{t_{0}, t_{1}, \ldots, t_{n-1}\right\}$ (where $n \geq 2$ ), and that $\mathcal{D}=\left\{t_{d}, \ldots, t_{n-1}\right\}$ (where $d \geq 1$ ).

Definition 2.6.

- By an $n$-sequent ${ }^{3}$ over a language $\mathcal{L}$ we mean an expression $\Sigma$ of the form $\Gamma_{0}\left|\Gamma_{1}\right| \ldots \mid \Gamma_{n-1}$, where, for each $i, \Gamma_{i}$ is a finite set of formulas of $\mathcal{L}$.

- A valuation $v$ satisfies the $n$-sequent $\Sigma=\Gamma_{0}\left|\Gamma_{1}\right| \ldots \mid \Gamma_{n-1}$, written $v \models \Sigma$, if there exists an $i, 0 \leq i \leq n-1$, and $\varphi_{i} \in \Gamma_{i}$ such that $v\left(\varphi_{i}\right)=t_{i}$.

- An n-sequent $\Sigma$ is valid in $\mathcal{V}$, in symbols $\models_{\mathcal{V}} \Sigma$, if $v \models \Sigma$ for every $v \in \mathcal{V}$.

To make the presentation more intuitive, from now on the sequent bar | which separates the non-designated values from the designated ones will usually be replaced with the symbol $\Rightarrow$ used in ordinary sequents.

As a simple consequence of the above we obtain:

Fact 1. A valuation $v \in \mathcal{V}$ satisfies an ordinary sequent $\Gamma \Rightarrow \Delta$ (see Definition 2.1 ) iff $v$ satisfies the $n$-sequent $\Gamma|\Gamma| \cdots|\Gamma \Rightarrow \Delta| \Delta|\ldots| \Delta$.

An alternative representation of $n$-sequents is provided by sets of $n$-signed formulas, shortly: signed formulas.

\section{Definition 2.7.}

- A signed formula over $\mathcal{L}$ and $\mathcal{T}$ is an expression of the form a: $\psi$, where $a \in \mathcal{T}, \psi \in \mathcal{W}$.

- A valuation $v$ in $\mathcal{V}$ satisfies a signed formula $a: \psi$, in symbols $v=a: \psi$, if $v(\psi)=a$.

Signed formulas will be denoted by $\alpha, \beta, \ldots$, and sets of signed formulas by $\Omega, \Sigma, \Phi$.

In the sequel, for any set $\mathcal{U} \subseteq \mathcal{T}$ of logical values and any set $F \subseteq \mathcal{W}$ of formulas of $\mathcal{L}$, we will also employ a shorthand notation of the form

$$
\mathcal{U}: F \stackrel{d f}{=}\{u: \varphi \mid u \in \mathcal{U}, \varphi \in F\}
$$

In terms of satisfaction by a valuation and validity, sets of signed formulas are interpreted disjunctively:

- A valuation $v \in \mathcal{V}$ satisfies a set of signed formulas $\Omega$ iff it satisfies some signed formula $\alpha \in \Omega$.

- A set of signed formulas $\Omega$ is valid, in symbols $\models \Omega$, iff $v \models \Omega$ for every valuation $v \in \mathcal{V}$.

The equivalence of the two formalisms follows from the following observation:

Fact 2. A sequent $\Sigma$ of the form $\Gamma_{0}\left|\Gamma_{1}\right| \ldots \mid \Gamma_{n-1}$ is valid if and only if the set $\Omega=\left\{t_{0}: \Gamma_{0}, t_{1}: \Gamma_{1}, \ldots, t_{n-1}: \Gamma_{n-1}\right\}$ of signed formulas is valid.

Accordingly, in future we will use both the representations alternately.

Assume $\mathcal{C}$ is an arbitrary $n$-sequent calculus for the language $\mathcal{L}$.

\footnotetext{
${ }^{3}$ Proof systems based on $n$-sequents were invented and reinvented several times in the past. See e.g. $[23,22,11]$. See also $[24,16,10]$ for further details and references.
} 
Definition 2.8. The consequence relation for $\mathcal{L}$ defined by $\mathcal{C}$ is the relation $\vdash_{\mathcal{C}}$ on sets of formulas of $\mathcal{W}$ such that, for any $T, S \subseteq W, T \vdash_{\mathcal{C}} S$ iff there exist finite subsets $\Gamma \subseteq T, \Delta \subseteq S$ such that the sequent $\Gamma|\Gamma| \ldots|\Gamma \Rightarrow \Delta| \Delta|\ldots| \Delta$ is provable in $\mathcal{C}$.

By Fact 2 we have that if $\mathcal{C}$ is sound and complete for $\mathcal{V}$ then:

$$
F \vdash_{\mathcal{C}} G \text { iff the set } \mathcal{N}: F \cup \mathcal{D}: G \text { is valid in } \mathcal{V}
$$

\subsection{Streamlining of Sequential Deduction Systems}

The $n$-sequent calculi obtained using the general method of [7] (and reviewed in the next subsection) are as a rule hardly optimal (as is usually the case with this type of "generic" systems). The same is true for the two-sided calculi which are obtained from them using the method described below. Therefore in both cases we use the three general streamlining principles from [7] to reduce them to a more compact form. Of these three, the first and the third decrease the number of rules (which is our main measure of complexity), while the second simplifies a rule by decreasing the number of its premises (since the third rule increases this number, its application is often followed by applications of the first two). Next we recall these three principles.

Denote the system under consideration by $\mathcal{R}$. Our streamlining principles consist in: deleting a derivable rule (Princ. 1), simplifying a rule by replacing it with one with weaker premises (Princ. 2), and combining two rules with the same conclusion (Princ. 3) into one.

Principle 1: If a rule in $\mathcal{R}$ is derivable from other rules, it can be deleted.

Principle 2: If $\frac{S}{\Sigma}$ (where $S$ is a set of premises) is a rule in $\mathcal{R}, S^{\prime}$ is a subset of $S$ and $\frac{S^{\prime}}{\Sigma}$ is derivable in $\mathcal{R}$ (perhaps using cuts), then $\frac{S}{\Sigma}$ can be replaced with $\frac{S^{\prime}}{\Sigma}$. In particular: if $\frac{S}{\Sigma}$ is a rule in $\mathcal{R}, \pi \in S$, and $\pi$ is derivable from $S \backslash\{\pi\}$ in $\mathcal{R}$, then $\frac{S}{\Sigma}$ can be replaced with $\frac{S \backslash\{\pi\}}{\Sigma}$ (two very simple, but quite useful cases of this are when $\pi$ is subsumed ${ }^{4}$ by an axiom or by some sequent in $S \backslash\{\pi\})$.

Principle 3: Two context-free rules ${ }^{5} \frac{\Omega_{1} \ldots \Omega_{k}}{\Sigma}$ and $\frac{\Omega_{1}^{\prime} \ldots \Omega_{l}^{\prime}}{\Sigma}$ can be replaced with the single rule $\frac{\left\{\Omega_{i} \cup \Omega_{j}^{\prime}\right\}_{1 \leq i \leq k, 1 \leq j \leq l}}{\Sigma}$

\footnotetext{
${ }^{4}$ On the propositional level, a sequent $\pi_{1}$ subsumes a sequent $\pi_{2}$ if the latter can be derived from the former using only weakenings.

${ }^{5} \mathrm{~A}$ rule $\mathrm{R}$ is context-free if whenever $\frac{\Phi_{1} \ldots \Phi_{k}}{\Sigma}$ is a valid application of $R$, and $\Sigma^{\prime}$ is a set of signed formulas, then $\frac{\Phi_{1} \cup \Sigma^{\prime} \ldots \Phi_{k} \cup \Sigma^{\prime}}{\Sigma \cup \Sigma^{\prime}}$ is also a valid application of R.
} 
The validity of Principles 1 and 2 is obvious. As for Principle 3 , since the second rule is context-free, for each $1 \leq i \leq k, \Omega_{i} \cup \Sigma$ follows from $\left.\left\{\Omega_{i} \cup \Omega_{j}^{\prime}\right\} \mid 1 \leq j \leq l\right\}$. But then $\Sigma$ follows from these $k$ sets using the first rule, since that rule is also context-free, and $\Sigma \cup \Sigma=\Sigma$.

A word is in order here about the role of the cut rule (a rule that can easily be generalized to n-sequents calculi). The rule should necessarily be at least admissible in any system which defines a consequence relation, in particular: in any system which is sound and complete for some reasonable semantics. It is indeed admissible in the n-sequent calculi from [7] described below. Now if the rule is admissible in a given ordinary sequent system then the same is true for the systems obtained from it by using our streamlining principles, even if cuts are used in applications of Principle II. Indeed, suppose $\Gamma \Rightarrow \Delta, \varphi$ and $\varphi, \Gamma \Rightarrow \Delta$ are both provable in the new system. Their proofs can then be turned into proofs with cuts in the old system. Since the cut rule is admissible in that system, both these sequents, as well as $\Gamma \Rightarrow \Delta$, have cut-free proofs in it. Now the cut-free proof of $\Gamma \Rightarrow \Delta$ in the old system can be turned into a cut-free proof of $\Gamma \Rightarrow \Delta$ in the new one simply by throwing away the appropriate parts of the original proof (i.e.: deleting superfluous premises of applications of the old rules, as well as the subproofs ending in them).

Note that the above argument does not work if the use of cuts is allowed in applications of Principle I.

Another important note: If a system is sound and complete for some semantics, then we may use a semantic version of Principle 2: If $\frac{S}{\Sigma}$ is a rule in $\mathcal{R}, S^{\prime}$ is a subset of $S$, and $\frac{S^{\prime}}{\Sigma}$ is sound for the semantics, then $\frac{S}{\Sigma}$ can be replaced with $\frac{S^{\prime}}{\Sigma}$. This version cannot be a part of a general simplification procedure, but there may be particular cases where it might be useful.

\section{5. $n$-sequent Systems for (Generalized) Finite-valued Logics}

In [7] we developed a generic $n$-sequent system (presented also as a RasiowaSikorski deduction system $([20,17])$ in a signed formula set form) for any logic based on an $n$-valued Nmatrix. That system, together with the method of translating an $n$-sequent calculus to an ordinary sequent calculus described in this paper, form the cornerstone of our method for producing cut-free two-sided sequent calculi for (generalized) finite-valued logics (and provide the basis for all the examples given below).

Given an $n$-valued Nmatrix $\mathcal{M}=(\mathcal{T}, \mathcal{D}, \mathcal{O})$, let $S F_{\mathcal{M}}$ be a deduction system defined as follows:

- Axioms: Each set of signed formulas of the form $\{a: \varphi \mid a \in \mathcal{T}\}$, where $\varphi$ is any formula in $\mathcal{W}$.

- Structural inference rules: Weakening. 
- Logical inference rules: For every $m$-ary connective $\diamond \in \mathcal{O}$ and any logical values $a_{1}, a_{2}, \ldots, a_{m}, b_{1}, b_{2}, \ldots, b_{k} \in \mathcal{T}$ such that $\widetilde{\diamond}\left(a_{1}, \ldots, a_{m}\right)=\left\{b_{1}, \ldots, b_{k}\right\}$, the rule:

$$
\frac{\Omega, a_{1}: \varphi_{1} \ldots \Omega, a_{m}: \varphi_{m}}{\Omega, b_{1}: \diamond\left(\varphi_{1}, \ldots, \varphi_{m}\right), \ldots, b_{k}: \diamond\left(\varphi_{1}, \ldots, \varphi_{m}\right)}
$$

Theorem 2.9. The system $S F_{\mathcal{M}}$ is sound and complete for the Nmatrix $\mathcal{M}$.

Note. In [7] we also provided an alternate semantics based on Nmatrices, the socalled static semantics, and developed two generic $n$-sequent calculi for it. The method we develop below applies to those calculi equally well.

\subsection{Paradigmatic Example: Rosser-Turquette Logics}

In [7] we exemplified the method of developing $n$-sequent system calculi introduced there, as well as the method of translating them to ordinary sequent calculi ${ }^{6}$, on several interesting and important logics. Now we shall give another, particularly suitable and illustrative example: the generic $n$-valued logics, where $n$ is any nonzero natural number, defined and examined by Rosser-Turquette [21]. The suitability of those logics for our purpose follows from their famous $J$ operators, being dichotomous "selectors" of the individual logical values. It is exactly those operators which make Rosser-Turquette logics sufficiently expressive to be eligible for our translation method, making them the simplest general example of the idea behind that method.

Rosser-Turquette logics can be seen as a general framework for reasoning about various degrees of truth, represented by the logical values $0,1, \ldots, n-1$, ordered linearly according to the order of natural numbers.

More exactly, the $n$-valued Rosser-Turquette logic $\mathcal{L}_{R T}$ can be represented by the ordinary matrix $\mathcal{M}_{R T}=(\mathcal{T}, \mathcal{D}, \mathcal{O})$, where $\mathcal{T}=\{0,1, \ldots, n-1\}, \mathcal{D}=\{s, s+$ $1, \ldots, n-1\}, \mathcal{O}=\left\{\neg, \vee, \wedge, J_{0}, J_{1}, \ldots, J_{n-1}\right\}$, and the connectives are interpreted as follows:

- $\widetilde{\neg} t= \begin{cases}n-1 & \text { if } t \in\{0, \ldots, s-1\}, \\ 0 & \text { otherwise. }\end{cases}$

- $t \widetilde{\mathrm{V}} u=\max (t, u) ; \quad t \widetilde{\wedge} u=\min (t, u)$;

- for $k=0,1, \ldots, n-1, \quad \widetilde{J_{k}} t= \begin{cases}n-1 & \text { if } t=k, \\ 0 & \text { otherwise; }\end{cases}$

A direct application of the well-known (see [16, 10]) general method for generating $n$-sequent calculi for ordinary matrices (of which the method described in Section 2.5 is a generalization) yields the system:

Axiom: $\{0: \varphi, 1: \varphi, \ldots,(n-1): \varphi\}$

\footnotetext{
${ }^{6}$ Note that the latter method was not defined in its general form there, but only illustrated on some examples.
} 
Logical inference rules:

$$
\begin{aligned}
& \text { ( }) \quad \frac{\Omega, k: \varphi \quad \Omega, l: \varphi}{\Omega, \max (k, l): \varphi \vee \psi} \quad(\wedge) \quad \frac{\Omega, k: \varphi \quad \Omega, l: \varphi}{\Omega, \min (k, l): \varphi \wedge \psi} \\
& (\neg 1) \quad \frac{\Omega, k: \varphi}{\Omega, n-1: \neg \varphi} \quad \text { for any } 0 \leq k \leq s-1 \\
& (\neg 2) \quad \frac{\Omega, k: \varphi}{\Omega, 0: \neg \varphi} \quad \text { for any } s \leq k \leq n-1 \\
& \left(\begin{array}{ll}
J_{k} 1
\end{array}\right) \frac{\Omega, k: \varphi}{\Omega, n-1: J_{k} \varphi} \quad\left(J_{k} 2\right) \frac{\Omega, l: \varphi}{\Omega, 0: J_{k}(\varphi)} \text { for } k \neq l
\end{aligned}
$$

After combining rules with the same conclusions using the streamlining Princ.3 above, we can replace the whole rule groups $(\neg 1),(\neg 2)$, and $\left(J_{k} 2\right)$ with the following single rules

$$
\begin{aligned}
& \left(\neg 1^{\prime}\right) \quad \frac{\Omega, 0: \varphi, 1: \varphi, \ldots, s-1: \varphi}{\Omega, n-1: \neg \varphi} \\
& \left(\neg 2^{\prime}\right) \quad \frac{\Omega, s: \varphi, s+1: \varphi, \ldots, n-1: \varphi}{\Omega, 0: \neg \varphi} \\
& \left(J_{k} 2^{\prime}\right) \quad \frac{\Omega,\{l: \varphi \mid l \neq k\}}{\Omega, 0: J_{k}(\varphi)}
\end{aligned}
$$

\section{Translation of $n$-sequent Calculi to Ordinary Ones}

In this section we show a general method of translating an $n$-sequent calculus for an $n$-valued logic satisfying certain expressiveness conditions to an ordinary, two-sided sequent calculus.

Below we use the notational conventions from the previous section. In addition, $\mathcal{F}_{1}$ will denote the set of formulas in $\mathcal{W}$ which have $p$ as their only propositional variable.

Definition 3.1. The language $\mathcal{L}$ is sufficiently expressive for the semantics $\mathcal{V}$ iff for any $i, 1 \leq i \leq n$, there exist natural numbers $l_{i}, m_{i} \geq 0$ and $A_{j}^{i}, B_{k}^{i} \in \mathcal{F}_{1}$, $1 \leq j \leq l_{i}, 1 \leq k \leq m_{i}$, such that, for any valuation $v \in \mathcal{V}$ and any formula $\varphi \in \mathcal{W}$, the following conditions are satisfied:

(i) $A_{1}^{i}=p$ for $i \in \mathcal{N}$ and $B_{1}^{i}=p$ for $i \in \mathcal{D}$;

(ii) For any $\varphi \in \mathcal{W}$ and any $t_{i} \in \mathcal{T}$

$$
v(\varphi)=t_{i} \Leftrightarrow v\left(A_{1}^{i} \varphi\right), \ldots, v\left(A_{l_{i}}^{i} \varphi\right) \in \mathcal{N} \& v\left(B_{1}^{i} \varphi\right), \ldots, v\left(B_{m_{i}}^{i} \varphi\right) \in \mathcal{D}
$$

where $A_{j}^{i} \varphi$ and $B_{k}^{i} \varphi$ denote the substitutions of $\varphi$ for $p$ in $A_{j}^{i}$ and $B_{k}^{i}$, respectively. 
Note that Condition (i) above is not really limiting, for, given $A_{j}^{i}$ 's, $B_{k}^{j}$, s satisfying (ii), we can simply add to them the necessary formula $p$ without violating (ii). Condition (i) will only be used for a backward translation from ordinary sequents to $n$-sequents, and will be disregarded otherwise.

The general transformation theorem will be based on replacing each $n$-sequent by a semantically equivalent set of two-sided sequents.

Definition 3.2. Let $\mathcal{L}$ be a sufficiently expressive language, with $l_{i}, m_{i}, A_{j}^{i}$ and $B_{k}^{i}$ like in Definition $3.1,{ }^{7}$ and let $\Sigma=\Gamma_{1}\left|\Gamma_{2}\right| \ldots \mid \Gamma_{n}$ be an $n$-sequent over $\mathcal{L}$. Call a partition sequence for $\Sigma$ any tuple $\pi=\left\langle\pi_{1}, \ldots, \pi_{n}\right\rangle$, where for $1 \leq i \leq n, \pi_{i}$ is a partition of $\Gamma_{i}$ of the form:

$$
\pi_{i}=\left\{\Gamma_{i j}^{\prime} \mid 1 \leq j \leq l_{i}\right\} \cup\left\{\Gamma_{i k}^{\prime \prime} \mid 1 \leq k \leq m_{i}\right\}
$$

For such a partition sequence $\pi$, and for $i=1,2, \ldots, n$, define:

$$
\begin{aligned}
& \Delta_{i}^{\prime}=\bigcup\left\{A_{j}^{i}\left(\Gamma_{i j}^{\prime}\right) \mid 1 \leq j \leq l_{i}\right\}, \quad \Delta_{i}^{\prime \prime}=\bigcup_{1}\left\{B_{k}^{i}\left(\Gamma_{i k}^{\prime \prime}\right) \mid 1 \leq k \leq m_{i}\right\} \\
& \Sigma_{\pi}=\Delta_{1}^{\prime}, \Delta_{2}^{\prime}, \ldots, \Delta_{n}^{\prime} \Rightarrow \Delta_{1}^{\prime \prime}, \Delta_{2}^{\prime \prime}, \ldots, \Delta_{n}^{\prime \prime}
\end{aligned}
$$

where $A_{j}^{i}\left(\Gamma_{i j}^{\prime}\right)=\left\{A_{j}^{i} \varphi \mid \varphi \in \Gamma_{i j}^{\prime}\right\}$, with $B_{k}^{i}\left(\Gamma_{i k}^{\prime \prime}\right)$ defined analogously. Denote by $\Pi$ the set of all partition sequences for $\Sigma$. Then by the set of two-sided sequents generated by $\Sigma$ we mean the set

$$
T W O(\Sigma)=\left\{\Sigma_{\pi} \mid \pi \in \Pi\right\}
$$

Note. By Condition (i) of Definition 3.1, $A_{1}^{i} \varphi=\varphi$ and $B_{1}^{i} \varphi=\varphi$. This implies that the sequent $\Gamma \Rightarrow \Delta$ is in $T W O(\Gamma|\Gamma| \ldots|\Gamma \Rightarrow \Delta| \Delta|\ldots| \Delta)$. Indeed, the sequent $\Sigma_{\pi}$ coincides with $\Gamma \Rightarrow \Delta$ when the partition sequence $\pi$ of the form (2) for the latter $n$-sequent is defined by: $\Gamma_{i 1}^{\prime}=\Gamma$ for $i<d, \Gamma_{i j}^{\prime}=\emptyset$ if $j>1$ or $i \geq d$, $\Gamma_{i 1}^{\prime \prime}=\Delta$ for $i \geq d$, and $\Gamma_{i k}^{\prime \prime}=\emptyset$ if $k>1$ or $i<d$.

Theorem 3.3. If $\mathcal{L}$ is a sufficiently expressive language, then, for any $n$-sequent $\Sigma=\Gamma_{1}\left|\Gamma_{2}\right| \ldots \mid \Gamma_{n}$ over $\mathcal{L}$ and any valuation $v \in \mathcal{V}$ of formulas in $\mathcal{L}$, $v$ satisfies $\Sigma$ iff $v$ satisfies all the two-sided sequents generated by $\Sigma$, i.e.

$$
v \models \Sigma \text { iff } v \models \Sigma^{\prime} \text { for every } \Sigma^{\prime} \in T W O(\Sigma)
$$

Proof. $(\Rightarrow)$ Assume $v \models \Sigma$. Then there exist $i, 1 \leq i \leq n$, and $\varphi \in \Gamma_{i}$ such that $v(\varphi)=t_{i}$. Hence by $(1)$ we have $v\left(A_{j}^{i} \varphi\right) \in \mathcal{N}$ for $j=1,2, \ldots, l_{i}$ and $v\left(B_{k}^{i} \varphi\right) \in \mathcal{D}$ for $k=1,2, \ldots, m_{i}$.

Now consider any two-sided sequent $\Sigma^{\prime} \in T W O(\Sigma)$. Then by (4) we have $\Sigma^{\prime}=\Sigma_{\pi}$ for some partition sequence $\pi \in \Pi$, where $\pi_{i}$ is of the form (2) (with $\Sigma_{\pi}$ defined as in (3)). Since $\varphi \in \Gamma_{i}$, and $\pi_{i}$ is a partition of $\Gamma_{i}$, then there must exist either $j$ such that $\varphi \in \Gamma_{i j}^{\prime}$ or $k$ such that $\varphi \in \Gamma_{i k}^{\prime \prime}$. In the first case, the left hand side of the sequent $\Sigma_{\pi}$ contains $A_{j}^{i} \varphi$, which in view of $v\left(A_{j}^{i} \varphi\right) \in \mathcal{N}$ implies

\footnotetext{
${ }^{7}$ Strictly speaking, all the concepts and notations introduced in this definition should be parametrized by the choice of $l_{i}, m_{i}, A_{j}^{i}$ and $B_{k}^{i}$. For the sake of readability, we omit this parametrization in the rest of this paper.
} 
$v \models \Sigma_{\pi}$. In the second case, the right hand side of the sequent $\Sigma_{\pi}$ contains $B_{k}^{i} \varphi$, which in view of $v\left(B_{k}^{i} \varphi\right) \in \mathcal{D}$ again implies $v \models \Sigma_{\pi}$.

$(\Leftarrow)$ Assume $v \models \Sigma^{\prime}$ for each $\Sigma^{\prime} \in T W O(\Sigma)$. Then by (4) $v \models \Sigma_{\pi}$ for each $\pi \in \Pi$. To prove that $v \models \Sigma$, we argue by contradiction. Suppose $v \not \models \Sigma$, i.e. $v(\varphi) \neq t_{i}$ for each $\varphi \in \Gamma_{i}, 1 \leq i \leq n$. In view of $(1)$, this means that

for any $1 \leq i \leq n$ and any $\varphi \in \Gamma_{i}$, either

(i) there exists $j, 1 \leq j \leq l_{i}$, such that $v\left(A_{j}^{i} \varphi\right) \notin \mathcal{N}$, or

(ii) there exists $k, 1 \leq k \leq m_{i}$, such that $v\left(B_{k}^{i} \varphi\right) \notin \mathcal{D}$

For any $i, 1 \leq i \leq n$ define:

$$
\begin{aligned}
& \Gamma_{i j}^{\prime}=\left\{\varphi \in \Gamma_{i} \mid v\left(A_{j}^{i} \varphi\right) \notin \mathcal{N}, \varphi \notin \bigcup_{t<j} \Gamma_{i t}^{\prime}\right\} \quad\left(1 \leq j \leq l_{i}\right) \\
& \Gamma_{i k}^{\prime \prime}=\left\{\varphi \in \Gamma_{i} \mid v\left(B_{k}^{i} \varphi\right) \notin \mathcal{D}, \varphi \notin \bigcup_{t<l_{i}} \Gamma_{i t}^{\prime} \cup \bigcup_{t<k} \Gamma_{i t}^{\prime \prime}\right\} \quad\left(1 \leq k \leq m_{i}\right)
\end{aligned}
$$

Let , $\pi=\left\langle\pi_{1}, \ldots, \pi_{n}\right\rangle$, where for each $i, 1 \leq i \leq n \pi_{i}$ is defined like in 2. By (6) and $(7), \pi$ is a partition sequence for $\Sigma$. In consequence, $\Sigma_{\pi}$ defined as in (3) is in $T W O(\Sigma)$. What is more, in view of (7), for any $\varphi \in \Gamma_{i j}^{\prime}$ we have $v\left(A_{j}^{i} \varphi\right) \in \mathcal{D}$, while for any $\varphi \in \Gamma_{i k}^{\prime \prime}$ we have $v\left(B_{k}^{i} \varphi\right) \in \mathcal{N}$. Thus $v \not \neq \Sigma_{\pi}$, which in view of $\Sigma_{\pi} \in T W O(\Sigma)$ is a contradiction.

The above theorem gives a clear way of translating a sound and complete $n$-sequent calculus to a sound and complete ordinary sequent calculus for $\mathcal{L}$.

Definition 3.4. Let $\mathcal{C}$ be an $n$-sequent calculus for the language $\mathcal{L}$. Then by $T W O(\mathcal{C})$ we mean the calculus of ordinary sequents for $\mathcal{L}$ consisting of:

- axioms: all two-sided sequents in $T W O(A)$, where $A$ is any axiom of $\mathcal{C}$

- inference rules: all rules of the form $\frac{T W O(S)}{\Sigma^{\prime}}$, where for some rule $\frac{S}{R}$ in $\mathcal{C}, \Sigma^{\prime} \in T W O(R)^{8}$.

Thus each $n$-sequent axiom is translated to the equivalent set of ordinary sequents, which now become axioms of the new ordinary sequent calculus. Further, given an $n$-sequent rule $\rho=\frac{S}{R}$, we translate each $n$-sequent $\Sigma$ in the premises $S$ and the conclusion $R$ to the equivalent set of ordinary sequents $T W O(\Sigma)$ defined above, obtaining the sets $S^{\prime}$ and $R^{\prime}$ of ordinary sequents, respectively (where $S^{\prime}$ is the union of the sets of ordinary sequents corresponding to the $n$-sequents in $S$ ). Then a rule $\rho$ is replaced by the equivalent set of ordinary sequent rules which allow us to derive each sequent in $R^{\prime}$ out of the sequents in $S^{\prime}$.

It should be noted that the axioms and rules obtained using the above general method are rather inefficient - in particular, the rules usually contain many superfluous premises. To remedy this, in practice we transform the resulting system into a simpler one, using the streamlining principles of Section 2.4.

${ }^{8}$ Here $T W O(S)=\bigcup\{T W O(\Sigma) \mid \Sigma \in S\}$ 
Theorem 3.5. If an $n$-sequent $\Sigma$ is provable in $\mathcal{C}$, then each ordinary sequent $\Sigma^{\prime} \in$ $T W O(\Sigma)$ is provable in $T W O(\mathcal{C})$.

Proof. By induction on the structure of the proof of $\Sigma$ in $\mathcal{C}$. If $\Sigma$ is an axiom of $\mathcal{C}$, then each sequent $\Sigma^{\prime} \in T W O(\Sigma)$ is an axiom of $T W O(\mathcal{C})$, so it has a trivial proof there. If $\Sigma$ is derived from $S$ using a rule $\frac{S}{\Sigma}$ in $\mathcal{C}$, then by induction hypothesis, the sequents in $T W O(\Delta)$ are provable in $T W O(\mathcal{C})$ for every $\Delta \in S$. In consequence, each sequent $\Sigma^{\prime} \in T W O(\Sigma)$ can be obtained from the above provable sequents using the rule $\frac{T W O(S)}{\Sigma^{\prime}}$ from $T W O(\mathcal{C})$. Accordingly, each such $\Sigma^{\prime}$ is provable in $T W O(\mathcal{C})$ too.

Theorem 3.6. Assume $\mathcal{L}$ is a sufficiently expressive language for $\mathcal{V}$, and $\mathcal{C}$ is a sound and complete sequent calculus for $\mathcal{V}$. Then $T W O(\mathcal{C})$ is a sound and complete ordinary sequent calculus for $\mathcal{V}$.

Proof. Soundness follows in an obvious way from the soundness of $\mathcal{C}$ and Theorem 3.3. Indeed, consider any rule $\rho^{\prime} \in T W O(C)$. Then for some rule $\rho=\frac{S}{R}$ in $\mathcal{C}, \rho^{\prime}=\frac{T W O(S)}{\Sigma^{\prime}}$, where $\Sigma^{\prime} \in T W O(R)$. As $\mathcal{C}$ is sound, then, for any $v, R$ is satisfied by $v$ whenever all the sequents in $S$ are satisfied by $v$. However, by Theorem 3.3, $v \models S$ iff $v \models \bigcup T W O(S)$. Thus $v \models T W O(S)$ implies $v \models S$, whence $v \models R$ by the soundness of $\rho$. But then $v \models \Sigma^{\prime}$ by Theorem 3.3 again, since $\Sigma^{\prime} \in T W O(R)$. Accordingly, $\rho^{\prime}$ is sound.

To prove completeness, assume a two-sided sequent $\Sigma^{\prime}=\Gamma \Rightarrow \Delta$ over $\mathcal{L}$ is valid. Then by Fact 1, the sequent $\Sigma=\Gamma|\Gamma| \ldots|\Gamma \Rightarrow \Delta| \Delta|\ldots| \Delta$ is also valid. As the calculus $\mathcal{C}$ is complete, $\Sigma$ must have a proof in $\mathcal{C}$. Since the sequent $\Sigma^{\prime}$ is in $T W O(\Sigma)$ by (i) of Definition 3.1, this proof can be translated to a proof of $\Sigma^{\prime}$ in $T W O(\mathcal{C})$ by Theorem 3.5 .

Corollary 3.7. Under the conditions of Theorem 3.6, the cut rule is admissible in $T W O(\mathcal{C})$ even if no rule in $\mathcal{C}$ induces it. In particular: if $\mathcal{C}$ is obtained by the method of [7] (described in 2.5 above), then the cut rule is admissible in $T W O(\mathcal{C})$.

Note. The proofs of Theorems 3.5 and 3.6 justify our claim in the introduction that our translation preserves the structures of proofs (in the sense explained there).

\section{Some Examples}

In this section, as well as in the following Applications section, we shall show how Theorems 3.3, 3.6 can be used to develop two-sided sequent calculi for certain many-valued logics. As part of the above, we shall also present more details regarding the examples on which the translation method was presented in [7]. 


\subsection{Rosser-Turquette Logics}

We begin with continuing the example of Rosser-Turquette $n$-valued logic from Section 2.6. As each $J_{k}$ operator is a dichotomous selector of the logical value $k$, then the language $\mathcal{L}_{R T}$ is sufficiently expressive. Indeed, as for any valuation $v$ over $\mathcal{M}_{R T}$, any formula $\varphi$ and any $k \in \mathcal{T}$ we have:

$$
v(\varphi)=k \text { iff } v\left(J_{k} \varphi\right) \in \mathcal{D}
$$

Namely, as $J_{k}: \mathcal{T} \rightarrow\{0, n-1\}$, then $v\left(J_{k} \varphi\right) \in \mathcal{D}$ iff $v\left(J_{k} \varphi\right)=n-1$, which holds iff $v(\varphi)=k$.

Hence $\mathcal{L}_{R T}$ satisfies Condition (1), and by the thesis of Theorem 3.3, for any valuation $v$ over $\mathcal{M}_{B}$, we have:

$$
v \models\left(\Gamma_{1}|\ldots| \Gamma_{s-1} \Rightarrow \Gamma_{s}|\ldots| \Gamma_{n-1}\right) \text { iff } v \models\left(\Rightarrow J_{0}\left(\Gamma_{0}\right), J_{1}\left(\Gamma_{1}\right), \ldots, J_{n-1}\left(\Gamma_{k}\right)\right)
$$

for any sets of formulas $\Gamma_{i}, i \in \mathcal{T}$.

Obviously, this makes the translation of the $n$-sequent calculus above into an ordinary calculus according to the procedure described in Theorems 3.3,3.6 especially simple, since the $J$ operators provide explicit separation of logical values using atomic formulas.. As a result, we obtain the following ordinary sequent calculus:

$$
\text { Axiom: } \Rightarrow J_{0} \varphi, J_{1} \varphi, \ldots, J_{n-1} \varphi
$$

Inference rules:

$$
\begin{array}{ll}
\frac{\Gamma \Rightarrow \Delta, J_{k} \varphi \quad \Gamma \Rightarrow \Delta, J_{l} \varphi}{\Gamma \Rightarrow \Delta, J_{\max (k, l)}(\varphi \vee \psi)} & \frac{\Gamma \Rightarrow \Delta, J_{k} \varphi \quad \Gamma \Rightarrow \Delta, J_{l} \varphi}{\Gamma \Rightarrow \Delta, J_{\min (k, l)}(\varphi \wedge \psi)} \\
\frac{\Gamma \Rightarrow \Delta, J_{0} \varphi, J_{1} \varphi, \ldots, J_{s-1}}{\Gamma \Rightarrow \Delta, J_{n-1} \neg \varphi} & \frac{\Gamma \Rightarrow \Delta, J_{s} \varphi, J_{s+1} \varphi, \ldots, J_{n-1} \varphi}{\Gamma \Rightarrow \Delta, J_{0} \neg \varphi} \\
\frac{\Gamma \Rightarrow \Delta, J_{k} \varphi}{\Gamma \Rightarrow \Delta, J_{n-1} J_{k} \varphi} & \frac{\Gamma \Rightarrow \Delta, J_{0} \varphi, \ldots, J_{k-1} \varphi, J_{k+1} \varphi, J_{n-1} \varphi}{\Gamma \Rightarrow \Delta, J_{0} J_{k} \varphi}
\end{array}
$$

\subsection{The logic $C_{\min }$}

In [7] we considered two 3 -valued Nmatrices $\mathcal{M}_{L}^{3}, \mathcal{M}_{S}^{3}$, for which the basic paraconsistent logic, usually denoted by $C_{\min }([14])$, is sound and complete.

For both the matrices we have $\mathcal{T}=\{\mathbf{f}, \top, \mathbf{t}\}, \mathcal{D}=\{\top, \mathbf{t}\}, \mathcal{O}=\{\widetilde{\neg}, \widetilde{V}, \widetilde{\wedge}, \widetilde{\supset}\}$, where the interpretations of $\vee, \wedge, \supset$ correspond in the obvious way to their interpretations in positive classical logic. For example, we have

$$
a \widetilde{\vee} b= \begin{cases}\mathcal{D} & \text { if either } a \in \mathcal{D} \text { or } b \in \mathcal{D} \\ \mathcal{N} & \text { if } a, b \in \mathcal{N}\end{cases}
$$

and analogously for $\widetilde{\wedge}, \widetilde{\supset}$. However, negation is interpreted differently in each of the matrices:

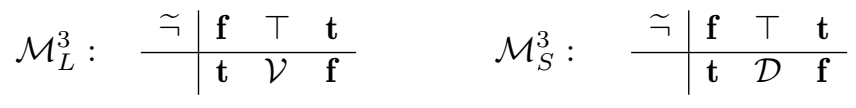


Accordingly, it can be easily seen that the language of $\mathcal{M}_{S}^{3}$ is sufficiently expressive in the sense of Definition 3.1, because

$$
\begin{array}{lll}
v(\varphi)=\mathbf{f} & \text { iff } & v(\varphi) \in \mathcal{N} \& v(\neg \varphi) \in \mathcal{D} \\
v(\varphi)=\top & \text { iff } & v(\varphi) \in \mathcal{D} \& v(\neg \varphi) \in \mathcal{D} \\
v(\varphi)=\mathbf{t} & \text { iff } & v(\varphi) \in \mathcal{D} \& v(\neg \varphi) \in \mathcal{N}
\end{array}
$$

However, the fact that $\widetilde{\neg}=\mathcal{T}$ in $\mathcal{M}_{L}^{3}$ makes it impossible to distinguish between $\top$ and $\mathbf{t}$, whence the language $\mathcal{M}_{L}^{3}$ is not sufficiently expressive for it. Hence just one of these matrices - namely, $\mathcal{M}_{S}^{3}$ - allows translation of the resulting calculus to an ordinary sequent calculus.

In [7] we obtained, using our general method, the following complete and cut-free 3 -sequent calculus for $\mathcal{M}_{S}^{3}$ :

Axiom: $(A) \varphi \Rightarrow \varphi \mid \varphi$

Inference rules:

$$
\begin{aligned}
& \frac{\Gamma_{\mathbf{f}}, \varphi \Rightarrow \Gamma_{\top}\left|\Gamma_{\mathbf{t}} \Gamma_{\mathbf{f}}, \psi \Rightarrow \Gamma_{\top}\right| \Gamma_{\mathbf{t}}}{\Gamma_{\mathbf{f}}, \varphi \vee \psi \Rightarrow \Gamma_{\top} \mid \Gamma_{\mathbf{t}}} \\
& \frac{\Gamma_{\mathbf{f}} \Rightarrow \Gamma_{\top}, \varphi, \psi \mid \Gamma_{\mathbf{t}}, \varphi, \psi}{\Gamma_{\mathbf{f}} \Rightarrow \Gamma_{\top}, \varphi \vee \psi \mid \Gamma_{\mathbf{t}}, \varphi \vee \psi} \\
& \frac{\Gamma_{\mathbf{f}}, \varphi, \psi \Rightarrow \Gamma_{\top} \mid \Gamma_{\mathbf{t}}}{\Gamma_{\mathbf{f}}, \varphi \wedge \psi \Rightarrow \Gamma_{\top} \mid \Gamma_{\mathbf{t}}} \\
& \frac{\Gamma_{\mathbf{f}} \Rightarrow \Gamma_{\top}, \varphi\left|\Gamma_{\mathbf{t}}, \varphi \quad \Gamma_{\mathbf{f}} \Rightarrow \Gamma_{\top}, \psi\right| \Gamma_{\mathbf{t}}, \psi}{\Gamma_{\mathbf{f}} \Rightarrow \Gamma_{\top}, \varphi \wedge \psi \mid \Gamma_{\mathbf{t}}, \varphi \wedge \psi} \\
& \frac{\Gamma_{\mathbf{f}} \Rightarrow \Gamma_{\top}, \varphi\left|\Gamma_{\mathbf{t}}, \varphi \Gamma_{\mathbf{f}}, \psi \Rightarrow \Gamma_{\top}\right| \Gamma_{\mathbf{t}}}{\Gamma_{\mathbf{f}}, \varphi \supset \psi \Rightarrow \Gamma_{\top} \mid \Gamma_{\mathbf{t}}} \\
& \frac{\Gamma_{\mathbf{f}}, \varphi \Rightarrow \Gamma_{\top}, \psi \mid \Gamma_{\mathbf{t}}, \psi}{\Gamma_{\mathbf{f}} \Rightarrow \Gamma_{\top}, \varphi \supset \psi \mid \Gamma_{\mathbf{t}}, \varphi \supset \psi} \\
& \frac{\Gamma_{\mathbf{f}} \Rightarrow \Gamma_{\top} \mid \Gamma_{\mathbf{t}}, \varphi}{\Gamma_{\mathbf{f}}, \neg \varphi \Rightarrow \Gamma_{\top} \mid \Gamma_{\mathbf{t}}} \\
& \frac{\Gamma_{\mathbf{f}}, \varphi \Rightarrow \Gamma_{\top} \mid \Gamma_{\mathbf{t}}}{\Gamma_{\mathbf{f}} \Rightarrow \Gamma_{\top} \mid \Gamma_{\mathbf{t}}, \neg \varphi} \\
& \frac{\Gamma_{\mathbf{f}} \Rightarrow \Gamma_{\top}, \varphi \mid \Gamma_{\mathbf{t}}}{\Gamma_{\mathbf{f}} \Rightarrow \Gamma_{\top}, \neg \varphi \mid \Gamma_{\mathbf{t}}, \neg \varphi}
\end{aligned}
$$

By Theorem 3.3, during the translation each 3-sequent $\Sigma=\Gamma_{\mathbf{f}} \Rightarrow \Gamma_{\top} \mid \Gamma_{\mathbf{t}}$ is replaced with the set $T W O(\Sigma)$ of all the ordinary sequents of the form

$$
\Gamma_{\mathbf{f}}^{\prime}, \neg \Gamma_{\mathbf{t}}^{\prime \prime} \Rightarrow \neg \Gamma_{\mathbf{f}}^{\prime \prime}, \Gamma_{\top}^{\prime}, \neg \Gamma_{\top}^{\prime \prime}, \Gamma_{\mathbf{t}}^{\prime}
$$

where $\Gamma_{i}=\Gamma_{i}^{\prime} \cup \Gamma_{i}^{\prime \prime}, \Gamma_{i}^{\prime} \cap \Gamma_{i}^{\prime \prime}=\emptyset$.

According to Theorem 3.6, to transform the above calculus to an ordinary one, we replace the axiom $A$ with the equivalent set of ordinary sequents $T W O(A)$, and each 3 -sequent rule $\rho=\frac{\mathcal{P}}{R}$ with the set of all ordinary sequent rules of the form $\frac{T W O(\mathcal{P})}{\Sigma^{\prime}}$, where $\Sigma^{\prime} \in T W O(R)$. Following that, the resulting calculus is reduced to a more efficient form using the streamlining principles.

Thus, the 3 -sequent axiom $A$ yields the ordinary sequents $\varphi \Rightarrow \varphi ; \neg \varphi, \varphi \Rightarrow \varphi ; \varphi \Rightarrow \neg \varphi, \varphi ; \neg \varphi, \varphi \Rightarrow \neg \varphi ; \Rightarrow \neg \varphi, \varphi ; \neg \varphi \Rightarrow \neg \varphi, \varphi ; \neg \varphi \Rightarrow \neg \varphi$ 
By deleting subsumed sequents ${ }^{9}$, this set can be reduced to just two axioms:

$$
(A 1) \varphi \Rightarrow \varphi, \quad(A 2) \Rightarrow \varphi, \neg \varphi
$$

To exemplify the method of translating the rules, consider first the second rule for disjunction. After removing the contexts for brevity, it takes the form:

$$
\frac{\Rightarrow \varphi, \psi \mid \varphi, \psi}{\Rightarrow \varphi \vee \psi \mid \varphi \vee \psi}
$$

From the premises we get the sequents

$$
\begin{array}{llll}
\Rightarrow \varphi, \psi & \neg \psi \Rightarrow \varphi, \psi & \neg \varphi \Rightarrow \varphi, \psi & \neg \varphi, \neg \psi \Rightarrow \varphi, \psi \\
\Rightarrow \varphi, \neg \psi, \psi & \neg \psi \Rightarrow \varphi, \neg \psi & \neg \varphi \Rightarrow \varphi, \neg \psi, \psi & \neg \varphi, \neg \psi \Rightarrow \varphi, \neg \psi \\
\Rightarrow \neg \varphi, \psi, \varphi & \neg \psi \Rightarrow \neg \varphi, \psi, \varphi & \neg \varphi \Rightarrow \neg \varphi, \psi & \neg \varphi, \neg \psi \Rightarrow \neg \varphi, \psi \\
\Rightarrow \neg \varphi, \neg \psi, \varphi, \psi & \neg \psi \Rightarrow \neg \varphi, \neg \psi, \varphi & \neg \varphi \Rightarrow \neg \varphi, \neg \psi, \psi & \neg \varphi, \neg \psi \Rightarrow \neg \varphi, \neg \psi
\end{array}
$$

After deleting the sequents subsumed by instances of Axioms $(A 1),(A 2)$ (using the streamlining Principle 2), we are left with

$$
\Rightarrow \varphi, \psi \quad \neg \psi \Rightarrow \varphi, \psi \quad \neg \varphi \Rightarrow \varphi, \psi \quad \neg \varphi, \neg \psi \Rightarrow \varphi, \psi
$$

Out of these sequents, the last three are subsumed by the first, leaving the single sequent $\Rightarrow \varphi, \psi$.

In turn, the conclusion yields the sequents

$$
\Rightarrow \varphi \vee \psi \quad \Rightarrow \neg(\varphi \vee \psi), \varphi \vee \psi \quad \neg(\varphi \vee \psi) \Rightarrow \varphi \vee \psi \quad \neg(\varphi \vee \psi) \Rightarrow \neg(\varphi \vee \psi)
$$

After deleting from this set the sequents which are subsumed by the first or by an instance of the axiom (A2) (using the streamlining Principle 1), the original 3-sequent rule is finally replaced by $\frac{\Rightarrow \varphi, \psi}{\Rightarrow \varphi \vee \psi}$, or, if we restore the contexts:

$$
\frac{\Gamma \Rightarrow \Delta, \varphi, \psi}{\Gamma \Rightarrow \Delta, \varphi \vee \psi}
$$

Next, let us consider the negation rules:

$$
(I) \frac{\Gamma_{\mathbf{f}} \Rightarrow \Gamma_{\top} \mid \Gamma_{\mathbf{t}}, \varphi}{\Gamma_{\mathbf{f}}, \neg \varphi \Rightarrow \Gamma_{\top} \mid \Gamma_{\mathbf{t}}} \quad(I I) \frac{\Gamma_{\mathbf{f}}, \varphi \Rightarrow \Gamma_{\top} \mid \Gamma_{\mathbf{t}}}{\Gamma_{\mathbf{f}} \Rightarrow \Gamma_{\top} \mid \Gamma_{\mathbf{t}}, \neg \varphi} \quad(I I I) \frac{\Gamma_{\mathbf{f}} \Rightarrow \Gamma_{\top}, \varphi \mid \Gamma_{\mathbf{t}}}{\Gamma_{\mathbf{f}} \Rightarrow \Gamma_{\top}, \neg \varphi \mid \Gamma_{\mathbf{t}}, \neg \varphi}
$$

In an informal notation, obtained by bunching together all the ordinary sequent rules derived from (I) by separating the alternate conclusion sequents with semicolons, from (I) we get (after skipping the usual contexts $\Gamma, \Delta$ )

$$
\begin{gathered}
\Rightarrow \varphi \neg \varphi \Rightarrow \\
\neg \varphi \Rightarrow ; \Rightarrow \neg \varphi
\end{gathered}
$$

As the first sequent below the line is identical with one of the premises, we can replace (I) by the rule

$$
(I-2) \frac{\Gamma \Rightarrow \Delta, \varphi \quad \Gamma, \neg \varphi \Rightarrow \Delta}{\Gamma \Rightarrow \Delta, \neg \neg \varphi}
$$

${ }^{9}$ This can be done using the streamlining Princ. 1 , since an axiom is just a rule with no premises. 
In turn, (II) yields $\frac{\varphi \Rightarrow \Rightarrow \neg \varphi}{\Rightarrow \neg \varphi ; \neg \neg \varphi \Rightarrow}$, generating the rule

$$
(I I-2) \frac{\Gamma, \varphi \Rightarrow \Delta \quad \Gamma \Rightarrow \Delta, \neg \varphi}{\Gamma, \neg \neg \varphi \Rightarrow \Delta}
$$

Finally, (III) yields

$$
\begin{gathered}
\Rightarrow \varphi \Rightarrow \neg \varphi \\
\Rightarrow \neg \varphi ; \neg \neg \varphi \Rightarrow \neg \varphi ; \neg \neg \varphi \Rightarrow \neg \neg \varphi ; \Rightarrow \neg \neg \varphi, \neg \varphi
\end{gathered}
$$

and generates no new rules, since three sequents under the line are derivable from the second premise using weakening, while the third one is axiomatic.

Next we can see that the first premise in rule (I-2) and the second premise in rule (II-2) are derivable from the other premise of the respective rule by cut with the excluded middle axiom $A 2: \Rightarrow \varphi, \neg \varphi$. Hence, by the streamlining Principle 2, the two rules can be simplified to:

$$
(I-2)^{*} \frac{\Gamma, \neg \varphi \Rightarrow \Delta}{\Gamma \Rightarrow \Delta, \neg \neg \varphi} \quad(I I-2)^{*} \frac{\Gamma, \varphi \Rightarrow \Delta}{\Gamma, \neg \neg \varphi \Rightarrow \Delta}
$$

Proceeding like this with the remaining rules, we obtain the standard sequent calculus for classical positive logic, augmented by the excluded middle axiom $A 2$, the restricted swap rule $(I-2)^{*}$ and the double negation rule $(I I-2)^{*}$.

Our general Theorem 2.9 (and the results of Section 3) ensure that the above system with axiom $A 2$ and rules $(I-2)^{*},(I I-2)^{*}$ is sound and complete, and that the cut rule is admissible in it. Let us denote this system by $S C_{\min }^{1}$. It is easy to see that both $(I-2)^{*}$ and axiom $A 2$ are derivable without cuts from the swap rule

$$
(S w) \frac{\Gamma, \varphi \Rightarrow \Delta}{\Gamma \Rightarrow \Delta, \neg \varphi}
$$

Now let let $S C_{\min }^{2}$ be the system obtained from $S C_{\min }^{1}$ by replacing rule $(I-2)^{*}$ and axiom $A 2$ by the swap rule $(S w)$. Then from our preceding considerations it follows that:

(i) Every (cut-free) proof of a sequent in $S C_{\min }^{1}$ can be transformed to a cut-free proof of the same sequent in $S C_{\min }^{2}$.

(ii) Since the swap rule is derivable in $S C_{\min }^{1}$ augmented by cut, every proof of a sequent in $S C_{\min }^{2}$ can be transformed to a proof with cuts of the same sequent in $S C_{\min }^{1}$. Since the weak cut-elimination theorem holds for $S C_{\min }^{1}$, in consequence, every sequent provable in $S C_{\min }^{2}$ has a cut-free proof in $S C_{\min }^{1}$.

From (i) and (ii) above it it follows that $S C_{\min }^{1}$ and $S C_{\min }^{2}$ are equivalent, and that the weak cut-elimination theorem holds for both of them ${ }^{10}$.

Observe that $S C_{\min }^{2}$ was not obtained from $S C_{\text {min }}^{1}$ using our streamlining principles (even though our considerations above are purely syntactic). Hence the

\footnotetext{
${ }^{10}$ Note that while the (weak) cut-elimination theorem for $S C_{\text {min }}^{2}$ directly follows from that of $S C_{\min }^{1}$ by (i), the converse is not true.
} 
two systems should be taken as different proof systems for $C_{\min }$. However, it is important to note that $S C_{\min }^{2}$ can be obtained directly by using another translation of our 3-sequents calculus: namely, one based on the simpler representation of the "sufficient expressiveness" condition which was used in [7]. In that representation, the first condition in (8) is replaced by

$$
v(\varphi)=\mathbf{f} \quad \text { iff } \quad v(\varphi) \in \mathcal{N}
$$

This gives rise to a different procedure for translating the original calculus. ${ }^{11}$

Finally, to understand better the points explained above, let $S C_{\min }^{3}$ be the system obtained from $S C_{\min }^{1}$ by deleting rule $(I-2)^{*}$ (or by replacing the swap rule in $S C_{\min }^{2}$ by the excluded middle axiom $A 2$ ). Since the swap rule (of which $(I-2)^{*}$ is a particular case) is derivable in $S C_{\min }^{3}$, the latter system is equivalent to $S C_{\min }^{1}$ (as well as to $S C_{\min }^{2}$ ). Consequently, it is sound and complete for $C_{\min }$. Now $S C_{\text {min }}^{3}$ is obviously simpler than $S C_{\min }^{1}$. However, we have not obtained it using our general method, and so there is no reason to believe that it is cut-free. Indeed it is not: it is easy to see that the sequent $\Rightarrow \varphi, \neg \neg \neg \varphi$ is valid in $C_{\min }$, and easily derivable in $S C_{\min }^{1}$ and in $S C_{\min }^{2}$, but has no cut-free proof in $S C_{\min }^{3}$. By the preceding considerations, it suffices to add to $S C_{\min }^{3}$ the special case $(I-2)^{*}$ of the swap rule to obtain a system for which weak cut-elimination holds (which is rather hard to guess in advance).

\subsection{Kleene and McCarthy logics}

Assume $\mathcal{T}=\{\mathbf{f}, \mathbf{e}, \mathbf{t}\}, \mathcal{D}=\{\mathbf{t}\}, \mathcal{O}=\{\widetilde{\sim}, \widetilde{V}, \underline{\mathbf{f}}, \underline{\mathbf{e}}, \underline{\mathbf{t}}\}$, and consider the Nmatrix $\mathcal{M}_{M K}=(\mathcal{T}, \mathcal{D}, \mathcal{O})$, where:

$$
\begin{array}{c|c|c|cc|c|c|c}
\widetilde{\sim} & \mathbf{f} & \mathbf{e} & \mathbf{t} \\
\hline & \mathbf{t} & \mathbf{e} & \mathbf{f}
\end{array} \quad \begin{array}{cc|c|c}
\widetilde{V} & \mathbf{f} & \mathbf{e} & \mathbf{t} \\
\hline \mathbf{f} & \mathbf{f} & \mathbf{e} & \mathbf{t} \\
\hline \mathbf{e} & \mathbf{e} & \mathbf{e} & \{\mathbf{e}, \mathbf{t}\} \\
\hline \mathbf{t} & \mathbf{t} & \mathbf{t} & \mathbf{t}
\end{array}
$$

with the constants $\underline{\mathbf{f}}, \underline{\mathbf{e}}, \underline{\mathbf{t}}$ interpreted in the obvious way.

In [7] we presented a sound and complete 3-sequent system for a static semantics of $\mathcal{M}_{M K}$ (which represents the intersection of the famous McCarthy and Kleene logics $[19,18])$. Now in $\mathcal{M}_{M K}$ the following holds for any $\varphi$ and $v$ :

$$
\begin{array}{llll}
v(\varphi)=\mathbf{t} & \text { iff } & v(\varphi) \in \mathcal{D} \\
v(\varphi)=\mathbf{e} & \text { iff } & v(\varphi) \in \mathcal{N} \& v(\neg \varphi) \in \mathcal{N} \\
v(\varphi)=\mathbf{f} & \text { iff } & v(\varphi) \in \mathcal{N} \& v(\neg \varphi) \in \mathcal{D}
\end{array}
$$

Since the static semantics of [7] (as a set of valuations) is a subset of the usual (dynamic) semantic induced by $\mathcal{M}_{M K}$, this implies that the language of $\mathcal{M}_{M K}$ is sufficiently expressive for its static semantics. It follows by Theorem 3.3 that a

\footnotetext{
${ }^{11}$ Note that this fact would directly imply that the cut elimination theorem holds for $S C_{\min }^{2}$. However, by itself it would not imply that this theorem holds also for $S C_{\min }^{1}$, but only that $S C_{\text {min }}^{1}$ augmented by cut is sound and complete.
} 
3-valued sequent $\Gamma_{\mathbf{f}} \mid \Gamma_{\mathbf{e}} \Rightarrow \Gamma_{\mathbf{t}}$ is satisfied by a valuation $v$ if and only if all the ordinary sequents of the form

$$
\Gamma_{\mathbf{f}}^{\prime}, \Gamma_{\mathbf{e}}^{\prime}, \neg \Gamma_{\mathbf{e}}^{\prime \prime} \Rightarrow \Gamma_{\mathbf{t}}, \neg \Gamma_{\mathbf{f}}^{\prime \prime}
$$

are satisfied by $v$, where $\Gamma_{i}=\Gamma_{i}^{\prime} \uplus \Gamma_{i}^{\prime \prime}$ for $i \in\{\mathbf{f}, \mathbf{e}\}$. Hence based on Theorem 3.6, we can translate the 3 -sequent calculus for $\mathcal{M}_{M K}$ to an ordinary, 2-sequent one. This calculus was described in [7] without giving many details on its derivation. Now we present two detailed examples how its rules have been derived.

As our first example we take the rule for introducing $\vee$ on the left hand side of the derived 2-sequent calculus. To obtain it, we start with the three rules of the 3-sequent calculus developed in [7] where disjunction is introduced in some component on the left hand side of $\Rightarrow$ :

$$
\begin{gathered}
\text { (1) } \frac{\Gamma_{\mathbf{f}}, \varphi\left|\Gamma_{\mathbf{e}} \Rightarrow \Gamma_{\mathbf{t}} \Gamma_{\mathbf{f}}, \psi\right| \Gamma_{\mathbf{e}} \Rightarrow \Gamma_{\mathbf{t}}}{\Gamma_{\mathbf{f}}, \varphi \vee \psi \mid \Gamma_{\mathbf{e}} \Rightarrow \Gamma_{\mathbf{t}}} \quad(2) \quad \frac{\Gamma_{\mathbf{f}}, \varphi\left|\Gamma_{\mathbf{e}} \Rightarrow \Gamma_{\mathbf{t}} \Gamma_{\mathbf{f}}\right| \Gamma_{\mathbf{e}}, \psi \Rightarrow \Gamma_{\mathbf{t}}}{\Gamma_{\mathbf{f}} \mid \Gamma_{\mathbf{e}}, \varphi \vee \psi \Rightarrow \Gamma_{\mathbf{t}}} \\
(3) \quad \frac{\Gamma_{\mathbf{f}}\left|\Gamma_{\mathbf{e}}, \varphi \Rightarrow \Gamma_{\mathbf{t}} \Gamma_{\mathbf{f}}, \psi\right| \Gamma_{\mathbf{e}}, \psi \Rightarrow \Gamma_{\mathbf{t}}}{\Gamma_{\mathbf{f}} \mid \Gamma_{\mathbf{e}}, \varphi \vee \psi \Rightarrow \Gamma_{\mathbf{t}}}
\end{gathered}
$$

Translating them into ordinary sequent rules and skipping the contexts for simplicity, we get the following three rules for introducing disjunction on the left hand side of $\Rightarrow$ :

$$
\begin{aligned}
& \frac{\varphi \Rightarrow \quad \Rightarrow \neg \varphi \quad \psi \Rightarrow \Rightarrow \neg \psi}{\varphi \vee \psi \Rightarrow} \\
& \frac{\varphi \Rightarrow \Rightarrow \neg \varphi \quad \psi \Rightarrow \neg \psi \Rightarrow}{\varphi \vee \psi \Rightarrow} \\
& \underline{\varphi \Rightarrow \quad \neg \varphi \Rightarrow \quad \psi \Rightarrow \quad\{\psi, \neg \psi \Rightarrow\} \quad\{\psi \Rightarrow \neg \psi\} \quad\{\neg \psi \Rightarrow \neg \psi\}} \underset{\varphi \vee \psi \Rightarrow}{ }
\end{aligned}
$$

In rule (3'), the last three premises, taken in braces, can be deleted, for the last of them is an axiom, and the first two are subsumed by $\psi \Rightarrow$.

Combining (1') and (2') with the help of Principle 3 from Section 2.4, we get a rule with 16 premises. However, it is easy to see that 12 of them are subsumed by either $\varphi \Rightarrow$ or $\Rightarrow \neg \varphi$ or $\psi \Rightarrow$, and another one, $\neg \psi \Rightarrow \neg \psi$, is an axiom. Thus we are left with the rule:

$$
\frac{\varphi \Rightarrow \quad \Rightarrow \neg \varphi \quad \psi \Rightarrow}{\varphi \vee \psi \Rightarrow}
$$

which we now combine with (3'), using the same Principle 3. After deleting premises subsumed by $\varphi \Rightarrow$ and $\psi \Rightarrow$ and the axiomatic premise $\neg \varphi \Rightarrow \neg \varphi$, we finally get the following well-known, simple rule:

$$
\frac{\Gamma, \varphi \Rightarrow \Delta \quad \Gamma, \psi \Rightarrow \Delta}{\Gamma, \varphi \vee \psi \Rightarrow \Delta}
$$


For our second example, we start from the following 3 -sequent rule from [7]:

$$
\frac{\Gamma_{\mathbf{f}}\left|\Gamma_{\mathbf{e}}, \varphi \Rightarrow \Gamma_{\mathbf{t}} \quad \Gamma_{\mathbf{f}}\right| \Gamma_{\mathbf{e}} \Rightarrow \Gamma_{\mathbf{t}}, \psi \quad \Gamma_{\mathbf{f}} \mid \Gamma_{\mathbf{e}} \Rightarrow \Gamma_{\mathbf{t}}, \underline{\mathbf{e}} \vee \underline{\mathbf{t}}}{\Gamma_{\mathbf{f}} \mid \Gamma_{\mathbf{e}} \Rightarrow \Gamma_{\mathbf{t}}, \varphi \vee \psi}
$$

A direct translation of it yields, after skipping the contexts, the ordinary rule:

$$
(*) \frac{\varphi \Rightarrow \neg \varphi \Rightarrow \quad \Rightarrow \psi \quad \Rightarrow \underline{\mathbf{e}} \vee \underline{\mathbf{t}}}{\Rightarrow \varphi \vee \psi}
$$

We next show that the first two premises of $(*)$ can be deleted. For this we first add $\varphi, \neg \varphi$ on the right hand side of all its premises and its conclusion. We get:

$$
\frac{\varphi \Rightarrow \varphi, \neg \varphi \quad \neg \varphi \Rightarrow \varphi, \neg \varphi \Rightarrow \psi, \varphi, \neg \varphi \Rightarrow \underline{\mathbf{e}} \vee \underline{\mathbf{t}}, \varphi, \neg \varphi}{(\Rightarrow \varphi \vee \psi), \varphi, \neg \varphi}
$$

The first two premises of this last rule can be skipped as axiomatic. As the other two can be derived from $\Rightarrow \psi$ and $\Rightarrow \underline{\mathbf{e}} \vee \underline{\mathbf{t}}$ (which are premises in $(*)$ ) by weakening, we get as a derived rule:

$$
\frac{\Gamma \Rightarrow \Delta, \psi \quad \Gamma \Rightarrow \Delta, \underline{\mathbf{e}} \vee \underline{\mathbf{t}}}{\Gamma \Rightarrow \Delta, \varphi \vee \psi, \varphi, \neg \varphi}
$$

Obviously, from the same two premises we can also infer $\Gamma \Rightarrow \Delta, \varphi, \psi$. Now from $\Gamma \Rightarrow \Delta, \varphi \vee \psi, \varphi, \neg \varphi$ and $\Gamma \Rightarrow \Delta, \varphi, \psi$ we can infer $\Gamma \Rightarrow \Delta, \varphi \vee \psi$ using the following rule (which also belongs to the set of rules for $\mathcal{M}_{M K}$ derived in [7]):

$$
\frac{\Gamma \Rightarrow \Delta, \varphi, \neg \varphi \quad \Gamma \Rightarrow \Delta, \varphi, \psi}{\Gamma \Rightarrow \Delta, \varphi \vee \psi}
$$

Hence in view of the streamlining Principle 2 we can indeed replace rule $(*)$ by:

$$
\frac{\Gamma \Rightarrow \Delta, \psi \quad \Gamma \Rightarrow \Delta, \underline{\mathbf{e}} \vee \underline{\mathbf{t}}}{\Gamma \Rightarrow \Delta, \varphi \vee \psi}
$$

We remind the reader that instead of the syntactic approach used above, we could have obtained the last rule from $(*)$ also by using a semantic approach, according to which we repeatedly check and eliminate from a rule premises which can be deleted without destroying the soundness of that rule.

\section{Application: Proof Systems for Information Sources Logics}

Now we will show that our method can be successfully applied to provide sequent calculi for a class of logics motivated by problems related to handling information coming from various sources, which are of fundamental importance in everyday practice. Since this application is new, we will use it to demonstrate in full our combined method for developing ordinary system calculi for generalized finitevalued logics, which couples the procedure for developing $n$-sequent calculi for such logics introduced in [7] with the method of translating them to ordinary calculi shown in the present paper. 
Suppose we have a framework for information collecting and processing, which consists a of set of information sources $S$ and a processor $P$. The sources provide information about formulas of classical logic $L_{C}$, and we assume that for each such formula $\varphi$, a source $s \in S$ can say that $\varphi$ is true, that $\varphi$ is false, or that it has no knowledge about $\varphi$. In turn, the processor collects information from the sources, combines it according to some strategy, and defines the resulting combined valuation of formulas in $L_{C}$. Clearly, for any formula $\varphi \in L_{C}$, the processor can encounter four possible situations:

- It has information that $\varphi$ is true but no information that $\varphi$ is false

- It has information that $\varphi$ is false but no information that $\varphi$ is true

- It has both information that $\varphi$ is true and information that $\varphi$ is false

- It has no information on $\varphi$ at all

In view of the above, a natural logical framework for this situation features four logical values corresponding to the four cases above, which we can denote by

$$
\mathbf{t}=\{1\}, \mathbf{f}=\{0\}, \top=\{0,1\}, \perp=\emptyset,
$$

Here 1 and 0 represent "true" and "false" (respectively), and so $\top$ represents inconsistent information, while $\perp$ absence of information. Among these four truth values we take as designated $\mathbf{t}$ and $T$ - the truth values whose assignment to a formula $\varphi$ means that the processor has information that $\varphi$ is true.

The above scenario has many ramifications, corresponding to various assumptions regarding the kind of information provided by the sources and the strategy used by the processor to combine it. Below we shall examine four main logics obtained under the assumption that the processor respects the deterministic consequences of the classical truth tables both ways. More exactly, this assumption means that the values assigned by the processor to complex formulas and those it assigns to their immediate subformulas are interrelated according to the following laws derived from the truth tables of classical logics:

(1): The processor ascribes 1 (true) to $\neg \varphi$ iff it it assigns 0 (false) to $\varphi$;

(2): The processor ascribes 0 to $\neg \varphi$ iff it assigns 1 to $\varphi$;

(3): If the processor ascribes 1 to either $\varphi$ or $\psi$, then it ascribes 1 to $\varphi \vee \psi$;

(4): The processor ascribes 0 to $\varphi \vee \psi$ iff it ascribes 0 to both $\varphi$ and $\psi$;

(5): The processor ascribes 1 to $\varphi \wedge \psi$ iff it assigns 1 to both $\varphi$ and $\psi$;

(6): If the processor ascribes 0 to either $\varphi$ or $\psi$, then it ascribes 0 to $\varphi \wedge \psi$.

Note that the converses of (3) and (6) do not hold. Note also that since the values of the resulting logic correspond to the subsets of $\{0,1\}$ ascribed to the formulas, the statement "the processor assigns 0 (1) to $\varphi$ " means that 0 (1) is included in the subset of $\{0,1\}$ being the value of $\varphi$.

Under the above assumptions, we can consider the following four scenarios:

1. CASE 1: the sources provide information about arbitrary formulas, both atomic and composed ones, but not necessarily about all of them.

2. CASE 2: as above, but the sources taken together are required to provide some information about all atomic formulas. 
3. CASE 3: the sources provide information about atomic formulas only. This information is then combined in a deterministic way to yield information about all formulas, including the composed ones.

4. CASE 4: like CASE 3, but, as in CASE 2, the sources taken together are required to provide some information about all atomic formulas.

Cases 3, 4 have already been considered in the past (see $[12,13]$ ) so we will not discuss them here, though it can be shown that their well-known proof systems can be derived with our methods. The new aspect contained in our approach is that the sources can also provide information about complex formulas. This is expressed by Cases 1, 2, which we shall examine in detail below.

\subsection{CASE 1}

We begin with the most general case, when the sources provide information about arbitrary formulas, also complex ones, but not necessarily all of them, so this information might be incomplete.

Rules (1)-(6) from the preceding section, which are obeyed by the processor in assigning values to formulas, imply that the presented setup can be described by a four-valued Nmatrix $\mathcal{M}_{I}^{4}=(\mathcal{T}, \mathcal{D}, \mathcal{O})$, where $\mathcal{T}=\{\mathbf{f}, \perp, \top, \mathbf{t}\}, \mathcal{D}=\{\top, \mathbf{t}\}, \mathcal{O}=$ $\{\widetilde{\neg}, \widetilde{V}, \widetilde{\wedge}\}$, and the non-deterministic interpretations of the connectives are given by the following tables:

\begin{tabular}{|c|c|c|c|c|c|c|c|c|c|c|c|c|}
\hline$\widetilde{V}$ & f & $\perp$ & $\top$ & t & & & & $\tilde{\Lambda}$ & f & $\perp$ & $\top$ & $\mathbf{t}$ \\
\hline$f$ & $\{\mathbf{f}, \top\}$ & $\{\mathbf{t}, \perp\}$ & $\{\top\}$ & $\{\mathbf{t}\}$ & & & & $f$ & $\{\mathbf{f}\}$ & $\{\mathbf{f}\}$ & $\{\mathbf{f}\}$ & $\{\mathbf{f}\}$ \\
\hline$\perp$ & $\{\mathbf{t}, \perp\}$ & $\{\mathbf{t}, \perp\}$ & $\{\mathbf{t}\}$ & $\{t\}$ & & & & $\perp$ & $\{\mathbf{f}\}$ & $\{\mathbf{f}, \perp\}$ & $\{\mathbf{f}\}$ & $\{\mathbf{f}, \perp\}$ \\
\hline T & $\{\top\}$ & $\{\mathbf{t}\}$ & $\{\top\}$ & $\{t\}$ & & & & $\top$ & $\{\mathbf{f}\}$ & $\{\mathbf{f}\}$ & $\{\top\}$ & $\{T\}$ \\
\hline $\mathbf{t}$ & $\{\mathbf{t}\}$ & $\{\mathbf{t}\}$ & $\{\mathbf{t}\}$ & $\{t\}$ & & & & $\mathbf{t}$ & $\{\mathbf{f}\}$ & $\{\mathbf{f}, \perp\}$ & $\{\top\}$ & $\{\mathbf{t}, \top\}$ \\
\hline & & & & $\widetilde{\neg}$ & f & $\perp$ & 1 & $\mathbf{I}$ & & & & \\
\hline & & & & & $\mathrm{t}$ & $\perp$ & 1 & f & & & & \\
\hline
\end{tabular}

We can see that the table for negation obeys rules (1), (2), the table for disjunction - rules (3), (4), and finally the table for conjunction - rules (5), (6). In other words, any valuation in the Nmatrix $\mathcal{M}_{I}^{4}$ represents possible information about values of formulas in the information sources-processor framework.

To see that is indeed so, let us first examine one of the most perplexing cases - the entry in the truth table for $\widetilde{\vee}$, saying that $\mathbf{f} \widetilde{\vee} \mathbf{f}=\{\mathbf{f}, \top\}$. Suppose $v(\varphi)=\mathbf{f}$, $v(\psi)=\mathbf{f}$. Then $0 \in v(\varphi)$ and $0 \in v(\psi)$, whence $0 \in v(\varphi \vee \psi)$ by Rule (4) above, based on the classical truth tables. If in addition one of the sources assigns 1 to $\varphi \vee \psi$, and consequently the processor ascribes 1 to $\varphi \vee \psi$ too, then $v(\varphi \vee \psi)=\top$, while otherwise $v(\varphi \vee \psi)=\mathbf{f}$. This justifies the two options included in the discussed table entry.

Another case worth considering is the entry in the same truth table saying that $\mathbf{f} \widetilde{\vee} \perp=\{\mathbf{t}, \perp\}$. Suppose $v(\varphi)=\mathbf{f}, v(\psi)=\perp$. Then $0 \notin v(\psi)$. By Rule (4) the processor cannot assign 0 to $\varphi \vee \psi$ in this case, because otherwise it has to assign 0 to $\psi$ too. However, it can assign 1 to $\varphi \vee \psi$ if one of the sources does so, for neither of the rules decrees that 1 must be assigned to either $\varphi$ or $\psi$ in that 
case. So the processor can assign either nothing or 1 to $\varphi \vee \psi$, whence it can only evaluate $v(\varphi \vee \psi)$ as either $\perp$ or $\mathbf{t}$, respectively.

5.1.1. A 4-sequent System for $\mathcal{M}_{I}^{4}$. We start with the system obtained from the generic system given in [7] and quoted in Section 2.5. Below we present its $\{\neg, \vee\}$ fragment. To make its presentation more compact, we use the signed formula set version of notation, where for brevity we often write $i: \varphi, \psi, \ldots$ instead of $i: \varphi, i: \psi, \ldots$.

Axiom: (A) f $: \varphi, \perp: \varphi, \top: \varphi, \mathbf{t}: \varphi$

Rules for Negation:
(N1) $\frac{\Omega, \mathbf{f}: \varphi}{\Omega, \mathbf{t}: \neg \varphi}$
(N2) $\frac{\Omega, \perp: \varphi}{\Omega, \perp: \neg \varphi}$
(N3) $\frac{\Omega, \top: \varphi}{\Omega, \top: \neg \varphi}$
$(N 4) \frac{\Omega, \mathbf{t}: \varphi}{\Omega, \mathbf{f}: \neg \varphi}$

Rules for Disjunction:
(D1) $\frac{\Omega, \mathbf{f}: \varphi \quad \Omega, \mathbf{f}: \psi}{\Omega, \mathbf{f}: \varphi \vee \psi, \top: \varphi \vee \psi}$
(D2) $\frac{\Omega, \mathbf{f}: \varphi \quad \Omega, \perp: \psi}{\Omega, \perp: \varphi \vee \psi, \mathbf{t}: \varphi \vee \psi}$
(D3) $\frac{\Omega, \mathbf{f}: \varphi \quad \Omega, \top: \psi}{\Omega, \top: \varphi \vee \psi}$
(D4) $\frac{\Omega, \tau: \varphi \quad \Omega, \mathbf{t}: \psi}{\Omega, \mathbf{t}: \varphi \vee \psi}$
$(D 5) \quad \frac{\Omega, \perp: \varphi \quad \Omega, \mathbf{f}: \psi}{\Omega, \perp: \varphi \vee \psi, \mathbf{t}: \varphi \vee \psi}$
(D6) $\frac{\Omega, \perp: \varphi \quad \Omega, \perp: \psi}{\Omega, \perp: \varphi \vee \psi, \mathbf{t}: \varphi \vee \psi}$
(D7) $\frac{\Omega, \perp: \varphi \quad \Omega, \top: \psi}{\Omega, \mathbf{t}: \varphi \vee \psi}$
$(D 8) \quad \frac{\Omega, \top: \varphi \quad \Omega, \mathbf{f}: \psi}{\Omega, \top: \varphi \vee \psi}$
(D9) $\frac{\Omega, \top: \varphi \quad \Omega, \perp: \psi}{\Omega, \mathbf{t}: \varphi \vee \psi}$
$(D 10) \frac{\Omega, \top: \varphi \quad \Omega, \top: \psi}{\Omega, \top: \varphi \vee \psi}$

$$
(D 11) \frac{\Omega, \mathbf{t}: \varphi \quad \Omega, \tau: \psi}{\Omega, \mathbf{t}: \varphi \vee \psi}
$$

Note that in the formulation of $(D 4)$ and $(D 11)$ we have employed a parameter $\tau$ representing an arbitrary logical value. Hence each of (D4) and (D11) is actually a rule schema representing a group of four rules of the same form. Now by using our streamlining Principle 3 (see Section 2.4) these four rules can be combined to a single rule with 16 premises. However, by deleting all the premises which are either axiomatic or subsumed by others, we remain in each case with a single premise: $\mathbf{t}: \psi$ in the case of $(D 4), \mathbf{t}: \varphi$ in the case of $(D 11)$. Finally, the resulting two rules can be combined to Rule $\left(D 4^{\prime}\right)$ below. 
By applying the same procedure to the other rules for disjunction, we can reduce Rules $(D 2)-(D 11)$ to the following four rules:

$$
\begin{aligned}
& \left(D 2^{\prime}\right) \frac{\Omega, \mathbf{f}: \varphi, \perp: \varphi \quad \Omega, \mathbf{f}: \psi, \perp: \psi \quad \Omega, \perp: \varphi, \psi}{\Omega, \perp: \varphi \vee \psi, \mathbf{t}: \varphi \vee \psi} \\
& \left(D 3^{\prime}\right) \frac{\Omega, \mathbf{f}: \varphi, \top: \varphi \quad \Omega, \mathbf{f}: \psi, \top: \psi \quad \Omega, \top: \varphi, \psi}{\Omega, \top: \varphi \vee \psi} \\
& \left(D 4^{\prime}\right) \quad \frac{\Omega, \mathbf{t}: \varphi, \psi}{\Omega, \mathbf{t}: \varphi \vee \psi} \quad\left(D 5^{\prime}\right) \frac{\Omega, \perp: \varphi, \psi \quad \Omega, \top: \varphi, \psi}{\Omega, \mathbf{t}: \varphi \vee \psi}
\end{aligned}
$$

where $\left(D 2^{\prime}\right)$ is obtained from $(D 2,5,6) ;\left(D 3^{\prime}\right)$ — from $(D 3,8,10)$; and $\left(D 5^{\prime}\right)$ from $(D 7,9)$. Out of them, Rules $\left(D 4^{\prime}\right)$ and $\left(D 5^{\prime}\right)$ can be still combined, yielding

$$
\left(D 4^{\prime \prime}\right) \frac{\Omega, \mathbf{t}: \varphi, \psi, \perp: \varphi, \psi \quad \Omega, \mathbf{t}: \varphi, \psi, \top: \varphi, \psi}{\Omega, \mathbf{t}: \varphi \vee \psi}
$$

All together, the original set of rules can be reduced to just four: $(D 1),\left(D 2^{\prime}\right)$, $\left(D 3^{\prime}\right),\left(D 4^{\prime \prime}\right)$. An analogous procedure yields the following rules for conjunction:

$$
\begin{aligned}
& (C 1) \quad \frac{\mathbf{f}: \varphi, \psi, \perp: \varphi, \psi \quad \mathbf{f}: \varphi, \psi, \top: \varphi, \psi}{\mathbf{f}: \varphi \wedge \psi} \\
& (C 2) \frac{\Omega, \perp: \varphi, \mathbf{t}: \varphi \quad \Omega, \perp: \psi, \mathbf{t}: \psi \quad \Omega, \perp: \varphi, \psi}{\Omega, \mathbf{f}: \varphi \wedge \psi, \perp: \varphi \wedge \psi} \\
& (C 3) \quad \frac{\Omega, \top: \varphi, \mathbf{t}: \varphi \quad \Omega, \top: \psi, \mathbf{t}: \psi \quad \Omega, \top: \varphi, \psi}{\Omega, \top: \varphi \wedge \psi} \\
& \text { (C4) } \frac{\Omega, \mathbf{t}: \varphi \quad \Omega, \mathbf{t}: \psi}{\Omega, \top: \varphi \wedge \psi, \mathbf{t}: \varphi \wedge \psi}
\end{aligned}
$$

5.1.2. Translation to Ordinary Sequent Calculus. Recall that the sets of nondesignated and designated values of our Nmatrix are $\mathcal{N}=\{\mathbf{f}, \perp\}$ and $\mathcal{D}=\{\top, \mathbf{t}\}$, respectively. Accordingly, it can be easily seen that the language $\mathcal{L}_{I}^{4}$ we are considering is sufficiently expressive for $\mathcal{M}_{I}^{4}$ in the sense of Definition 3.1, since, for any valuation $v$ and any formula $\varphi$, we have:

$$
\begin{array}{lllll}
v(\varphi)=\mathbf{f} & \Leftrightarrow & v(\varphi) \in \mathcal{N} & \& & v(\neg \varphi) \in \mathcal{D} \\
v(\varphi)=\perp & \Leftrightarrow & v(\varphi) \in \mathcal{N} & \& & v(\neg \varphi) \in \mathcal{N} \\
v(\varphi)=\top & \Leftrightarrow & v(\varphi) \in \mathcal{D} & \& & v(\neg \varphi) \in \mathcal{D} \\
v(\varphi)=\mathbf{t} & \Leftrightarrow & v(\varphi) \in \mathcal{D} & \& & v(\neg \varphi) \in \mathcal{N}
\end{array}
$$

By Theorem 3.3, this implies that a 4-sequent $\Gamma_{\mathbf{f}}\left|\Gamma_{\perp} \Rightarrow \Gamma_{\top}\right| \Gamma_{\mathbf{t}}$ - in our current notation, $\mathbf{f}: \Gamma_{\mathbf{f}}, \perp: \Gamma_{\perp}, \top: \Gamma_{\top}, \mathbf{t}: \Gamma_{\mathbf{t}}-$ is valid if and only if so are all the elements of the set $T W O(\Sigma)$ of all the ordinary sequents of the form

$$
\Gamma_{\mathbf{f}^{\prime}}, \Gamma_{\perp}^{\prime}, \neg \Gamma_{\perp}^{\prime \prime}, \neg \Gamma_{\mathbf{t}}^{\prime \prime} \Rightarrow \neg \Gamma_{\mathbf{f}}^{\prime \prime}, \Gamma_{\top}^{\prime}, \neg \Gamma_{\top}^{\prime \prime}, \Gamma_{\mathbf{t}}^{\prime}
$$


where $\Gamma_{i}=\Gamma_{i}^{\prime} \cup \Gamma_{i}^{\prime \prime}, \Gamma_{i}^{\prime} \cap \Gamma_{i}^{\prime \prime}=\emptyset$

Let us exemplify the translation to an ordinary sequent calculus following from (11) and Theorem 3.6 on the axiom of our system, and on Rule (D1). The set of ordinary sequents equivalent to the 4-sequent axiom under (11) is:

$\{\varphi \Rightarrow \varphi ; \neg \varphi \Rightarrow \neg \varphi ; \varphi, \neg \varphi \Rightarrow \varphi ; \varphi \Rightarrow \neg \varphi, \varphi ; \neg \varphi \Rightarrow \neg \varphi, \varphi ; \varphi, \neg \varphi \Rightarrow \varphi ; \varphi, \neg \varphi \Rightarrow \varphi, \neg \varphi\}$

Since all the sequents in this set are subsumed by the first, we can take the usual basic axiom $\psi \Rightarrow \psi$ as the only axiom.

Let us now pass to Rule $(D 1)$. In the standard 4-sequent notation used in the translation theorem and in (11), this rule takes the form:

$$
\frac{\Gamma_{\mathbf{f}}, \varphi\left|\Gamma_{\perp} \Rightarrow \Gamma_{\top}\right| \Gamma_{\mathbf{t}} \quad \Gamma_{\mathbf{f}}, \psi\left|\Gamma_{\perp} \Rightarrow \Gamma_{\top}\right| \Gamma_{\mathbf{t}}}{\Gamma_{\mathbf{f}}, \varphi \vee \psi\left|\Gamma_{\perp} \Rightarrow \Gamma_{\top}, \varphi \vee \psi\right| \Gamma_{\mathbf{t}}}
$$

Hence, if we replace the arbitrary context in the 4-sequents above by an arbitrary context $\Gamma, \Delta$ in two-sided sequents, the set of ordinary sequents corresponding to the premises under (11) can be written as

$$
P^{\prime}=\{\Gamma, \varphi \Rightarrow \Delta ; \quad \Gamma \Rightarrow \Delta, \neg \varphi ; \quad \Gamma, \psi \Rightarrow, \Delta ; \quad \Gamma \Rightarrow \Delta, \neg \psi\}
$$

while the set of ordinary sequents corresponding to the conclusion is

$$
\begin{aligned}
R^{\prime}= & \{\Gamma, \varphi \vee \psi \Rightarrow \Delta, \varphi \vee \psi ; \quad \Gamma, \varphi \vee \psi \Rightarrow \Delta, \neg(\varphi \vee \psi) ; \\
& \Gamma \Rightarrow \Delta, \neg(\varphi \vee \psi) ; \quad \Gamma \Rightarrow \Delta, \varphi \vee \psi, \neg(\varphi \vee \psi)\}
\end{aligned}
$$

As the first sequent in $R^{\prime}$ is an instance of the axiom of the ordinary sequent calculus, and all others are subsumed by $\Gamma \Rightarrow \Delta, \neg(\varphi \vee \psi)$, we are only left with the latter one, yielding the rule

$$
\frac{\Gamma, \varphi \Rightarrow \Delta ; \quad \Gamma \Rightarrow \Delta, \neg \varphi ; \quad \Gamma, \psi \Rightarrow, \Delta ; \quad \Gamma \Rightarrow \Delta, \neg \psi}{\Gamma \Rightarrow \Delta, \neg(\varphi \vee \psi)}
$$

It can be shown that using our streamlining principles this last rule can be simplified by eliminating its first and third premises ${ }^{12}$. We thus finally obtain:

$$
\frac{\Gamma \Rightarrow \Delta, \neg \varphi \quad \Gamma \Rightarrow \Delta, \neg \psi}{\Gamma \Rightarrow \Delta, \neg(\varphi \vee \psi)}
$$

Applying same procedure to all the remaining rules, we finally translate our 4-valued calculus into the following ordinary calculus:

Axiom: $\varphi \Rightarrow \varphi$

\footnotetext{
${ }^{12}$ Again, we can instead use the semantic approach to check that the rule remains valid for $\mathcal{M}_{I}^{4}$ after eliminating these premises.
} 


\section{Inference rules:}
(n1) $\frac{\Gamma, \varphi \Rightarrow \Delta}{\Gamma, \neg \neg \varphi \Rightarrow \Delta}$
(n2) $\frac{\Gamma \Rightarrow \Delta, \varphi}{\Gamma \Rightarrow \Delta, \neg \neg \varphi}$
(d1) $\frac{\Gamma \Rightarrow \Delta, \varphi, \psi}{\Gamma \Rightarrow \Delta, \varphi \vee \psi}$
(d2) $\frac{\Gamma, \neg \varphi, \neg \psi \Rightarrow \Delta}{\Gamma, \neg(\varphi \vee \psi) \Rightarrow \Delta}$
(d3) $\frac{\Gamma \Rightarrow \Delta, \neg \varphi \quad \Gamma \Rightarrow \Delta, \neg \psi}{\Gamma \Rightarrow \Delta, \neg(\varphi \vee \psi)}$
(c1) $\frac{\Gamma, \varphi, \psi \Rightarrow \Delta,}{\Gamma, \varphi \wedge \psi \Rightarrow \Delta}$
(c2) $\frac{\Gamma \Rightarrow \Delta, \neg \varphi, \neg \psi}{\Gamma \Rightarrow \Delta, \neg(\varphi \wedge \psi)}$
(c3) $\frac{\Gamma \Rightarrow \Delta, \varphi \quad \Gamma \Rightarrow \Delta, \psi}{\Gamma \Rightarrow \Delta, \varphi \wedge \psi}$

Therefore from our general theorems we get:

Theorem 5.1. The ordinary sequent calculus developed above is sound and complete for $\mathcal{M}_{I}^{4}$, and the cut rule is admissible in it.

\subsection{CASE 2}

The second case is when the sources provide complete information about all atomic formulas. Thus, for any atomic formula $p$ of the classical language $\mathcal{L}_{C}$, some source in $S$ must say either that $p$ is true or that $p$ is false. Assuming the general rules for the processor's behavior given in the introduction to Section 5 , one can easily prove by induction that under this condition no formula is given the value $\perp$. Hence this scenario gives rise to a logic based on the three-valued Nmatrix $\mathcal{M}_{I}^{3}=(\mathcal{T}, \mathcal{D}, \mathcal{O})$, where $\mathcal{T}=\{\mathbf{f}, \top, \mathbf{t}\}, \mathcal{D}=\{\top, \mathbf{t}\}, \mathcal{O}=\{\widetilde{\neg}, \widetilde{V}, \widetilde{\wedge}\}$, and the non-deterministic interpretations of the connectives are given by:

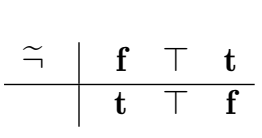

\begin{tabular}{c|ccc}
$\widetilde{V}$ & $\mathbf{f}$ & $\top$ & $\mathbf{t}$ \\
\hline $\mathbf{f}$ & $\{\mathbf{f}, \top\}$ & $\{\top\}$ & $\{\mathbf{t}\}$ \\
$\top$ & $\{\top\}$ & $\{\top\}$ & $\{\mathbf{t}\}$ \\
$\mathbf{t}$ & $\{\mathbf{t}\}$ & $\{\mathbf{t}\}$ & $\{\mathbf{t}\}$
\end{tabular}

\begin{tabular}{c|ccc}
$\widetilde{\wedge}$ & $\mathbf{f}$ & $\top$ & $\mathbf{t}$ \\
\hline $\mathbf{f}$ & $\{\mathbf{f}\}$ & $\{\mathbf{f}\}$ & $\{\mathbf{f}\}$ \\
$\top$ & $\{\mathbf{f}\}$ & $\{\top\}$ & $\{\top\}$ \\
$\mathbf{t}$ & $\{\mathbf{f}\}$ & $\{\top\}$ & $\{\mathbf{t}, \top\}$
\end{tabular}

Again we can first develop a 3-sequent system for the logic under consideration by using the general method from [7]. What we obtain after applying the streamlining principles are the axiom and rules shown below:

Axiom: $\mathbf{f}: \varphi, \top: \varphi, \mathbf{t}: \varphi$

\section{Inference rules:}

\section{Negation:}
$(N 1) \frac{\Omega, \mathbf{f}: \varphi}{\Omega, \mathbf{t}: \neg \varphi}$
(N2) $\frac{\Omega, \top: \varphi}{\Omega, \top: \neg \varphi}$
$(N 3) \frac{\Omega, \mathbf{t}: \varphi}{\Omega, \mathbf{f}: \neg \varphi}$ 


\section{Disjunction:}

$$
\begin{array}{ll}
\text { (D1) } & \frac{\Omega, \mathbf{f}: \varphi \quad \Omega, \mathbf{f}: \psi}{\Omega, \mathbf{f}: \varphi \vee \psi, \top: \varphi \vee \psi} \\
(D 2) & \frac{\Omega, \mathbf{f}: \varphi, \top: \varphi \quad \Omega, \mathbf{f}: \psi, \top: \psi \quad \Omega, \top: \varphi, \psi}{\Omega, \top: \varphi \vee \psi} \\
\text { (D3) } & \frac{\Omega, \mathbf{t}: \varphi, \psi}{\Omega, \mathbf{t}: \varphi \vee \psi}
\end{array}
$$

\section{Conjunction:}

$$
\begin{array}{ll}
(C 1) & \frac{\Omega, \mathbf{f}: \varphi, \psi}{\Omega, \mathbf{f}: \varphi \wedge \psi} \\
(C 2) & \frac{\Omega, \top: \varphi, \mathbf{t}: \varphi \quad \Omega, \top: \psi, \mathbf{t}: \psi \quad \Omega, \top: \varphi, \psi}{\Omega, \top: \varphi \wedge \psi} \\
(C 3) \quad \frac{\Omega, \mathbf{t}: \varphi \quad \Omega, \mathbf{t}: \psi}{\Omega, \top: \varphi \wedge \psi, \mathbf{t}: \varphi \wedge \psi} &
\end{array}
$$

As the sets of non-designated and designated values of our Nmatrix are $\mathcal{N}=\{\mathbf{f}\}$ and $\mathcal{D}=\{\top, \mathbf{t}\}$, respectively, it can be easily seen that the language $\mathcal{L}_{I}^{3}$ we are considering is sufficiently expressive for $\mathcal{M}_{I}^{3}$. Indeed, for any valuation $v$ and any formula $\varphi$, we have:

$$
\begin{array}{lllll}
v(\varphi)=\mathbf{f} & \Leftrightarrow & v(\varphi) \in \mathcal{N} & \& & v(\neg \varphi) \in \mathcal{D} \\
v(\varphi)=\top & \Leftrightarrow & v(\varphi) \in \mathcal{D} & \& & v(\neg \varphi) \in \mathcal{D} \\
v(\varphi)=\mathbf{t} & \Leftrightarrow & v(\varphi) \in \mathcal{D} & \& & v(\neg \varphi) \in \mathcal{N}
\end{array}
$$

By Theorem 3.3, this implies that a 3-sequent $\Gamma_{\mathbf{f}} \Rightarrow \Gamma_{\top} \mid \Gamma_{\mathbf{t}}$ - in our current notation, $\mathbf{f}: \Gamma_{\mathbf{f}}, \top: \Gamma_{\top}, \mathbf{t}: \Gamma_{\mathbf{t}}$ - is valid if and only if so are all the ordinary sequents of the form $\Gamma_{\mathbf{f}}^{\prime}, \neg \Gamma_{\mathbf{t}}^{\prime \prime} \Rightarrow \neg \Gamma_{\mathbf{f}}^{\prime \prime}, \Gamma_{T}^{\prime}, \neg \Gamma_{T}^{\prime \prime}, \Gamma_{\mathbf{t}}^{\prime}$, where $\Gamma_{\tau}=\Gamma_{\tau}^{\prime} \uplus \Gamma_{\tau}^{\prime \prime}$. Therefore with the help of Theorem 3.6 we can translate the above 3 -sequent calculus to the ordinary sequent calculus we have obtained in the preceding section for $\mathcal{M}_{I}^{4}$, augmented with the excluded middle axiom $\Rightarrow \varphi, \neg \varphi$.

Alternatively, since now $\mathcal{N}=\{\mathbf{f}\}$, the first line of (12) can be simplified to

$$
v(\varphi)=\mathbf{t} \text { iff } v(\varphi) \in \mathcal{N}
$$

The translation which is based on this simplified form of (12) again leads us to a different (though of course equivalent) ordinary sequent calculus. Namely, we get just the single basic axiom $\varphi \Rightarrow \varphi$, while the excluded middle axiom is replaced by the left-to-right swap rule. Thus the new negation rules are:

$$
\frac{\Gamma \Rightarrow \Delta, \varphi}{\Gamma, \neg \neg \varphi \Rightarrow \Delta} \quad \frac{\Gamma \Rightarrow \Delta, \varphi}{\Gamma \Rightarrow \Delta, \neg \neg \varphi} \quad \frac{\Gamma, \varphi \Rightarrow \Delta}{\Gamma \Rightarrow \Delta, \neg \varphi}
$$


while all the disjunction and conjunction rules remain unchanged. Thus the situation here is similar to that in the case of $C_{\min }$ considered above. However, here the addition of the swap rule does not allow us to eliminate any of the previous negation rules, for none of them is derivable from it.

\section{References}

[1] A. Avron, Non-deterministic Semantics for Families of Paraconsistent Logics, To appear in Paraconsistency with no Frontiers (J.-Y. Beziau and W. Carnielli, eds.).

[2] A. Avron, A Non-deterministic View on Nonclassical Negations, Studia Logica 80, 159-194 (2005).

[3] A. Avron, Non-deterministic Matrices and Modular Semantics of Rules, in Logica Universalis (J.-Y. Beziau, ed.), 149-167, Birkhäuser Verlag, 2005.

[4] A. Avron, Non-deterministic Semantics for Paraconsistent C-systems, in Proceedings of 8th ECSQARU (Euro. Conf. Symb. and Quant. Approaches to Reasoning on Uncertainty) (L. Godo, ed.), 625-637, LNAI 3571, Springer, 2005.

[5] A. Avron, Logical Non-determinism as a Tool for Logical Modularity: An Introduction, in We Will Show Them: Essays in Honour of Dov Gabbay, Vol 1 (S. Artemov, H. Barringer, A. S. d'Avila Garcez, L. C. Lamb, and J. Woods, eds.), 105-124, College Publications, 2005.

[6] A. Avron, Non-deterministic Semantics for Logics with a Consistency Operators, Forthcoming in the International Journal of Approximate Reasoning.

[7] A. Avron and B. Konikowska, Multi-valued Calculi for Logics Based on Nondeterminism, Proceedings COS'04 (Challenge of Semantics Workshop), Vienna 2004, Journal of the Interest Group in Pure and Applied Logic, 2005 (10), pp. 365-387.

[8] A. Avron and I. Lev, Canonical Propositional Gentzen-Type Systems, in Proceedings of the 1st International Joint Conference on Automated Reasoning (IJCAR 2001) (R. Goré, A Leitsch, T. Nipkow, Eds), LNAI 2083, 529-544, Springer Verlag, 2001.

[9] A. Avron, and I. Lev, Non-deterministic Multiple-valued Structures, Journal of Logic and Computation, Vol. 15 (2005), 241-261.

[10] M. Baaz, C. G. Fermüller, and G. Salzer, Automated Deduction for Many-valued Logics, in Handbook of Automated Reasoning (Robinson, A. and Voronkov, A. eds.), 1355-1400, Elsevier Science Publishers, 2000.

[11] M. Baaz, C. G. Fermüller, and R. Zach, Elimination of Cuts in First-order Finitevalued Logics, Information Processing Cybernetics 29 (1994), 333-355. Reports on Mathematical Logic 33 (1999), 29-44.

[12] N. D. Belnap, A Useful Four-valued Logic, in Modern Uses of Multiple-Valued Logic (G. Epstein and J. M. Dunn, eds), 7-37, Reidel, 1977.

[13] W. A. Carnielli and M. Lima.-Marques, Society semantics for multiple-valued logics, in Proceedings of the XII EBL- Advances in Contemporary Logic and Computer Science (Walter A. Carnielli and Itala M. L. D'Ottaviano, eds.), American Mathematical Society, Series Contemporary Mathematics, Volume 235, 33-52, 1999. 
[14] W. A. Carnielli and J. Marcos, A Taxonomy of C-systems, in Paraconsistency — the logical way to the inconsistent (W. A. Carnielli, M. E. Coniglio, I. L. M. D'ottaviano, eds.), Lecture Notes in Pure and Applied Mathematics, 1-99, Marcell Dekker, 2002.

[15] W. A. Carnielli, M. E. Coniglio, J. Marcos, Logics of Formal Inconsistency, forthcoming Handbook of Philosophical Logic, 2nd edition (D. Gabbay and F. Guenthner, eds), Kluwer Academic Publishers.

[16] R. Hähnle, Tableaux for Multiple-valued Logics, in Handbook of Tableau Methods (D’Agostino, M., Gabbay, D.M., Hähnle, R. and Posegga, J. eds.), 529-580, Kluwer Publishing Company, 1999.

[17] B. Konikowska, Rasiowa-Sikorski Deduction Systems in Computer Science Applications, Theoretical Computer Science 286 (2002), pp. 323-266.

[18] B. Konikowska, A. Tarlecki, A. Blikle, A Three-valued Logic for Software Specification and Validation, Fundam. Inform. 14(4), 411-453, 1991.

[19] J. McCarthy, A Basis For a Mathematical Theory of Computation, Western Joint Conference, 1961, later published in Computer Programming and Formal Systems, North Holland 1967, 33-70,

[20] H. Rasiowa and R. Sikorski, The Mathematics of Metamathematics, PWN (Polish Scientific Publishers), Warsaw 1963.

[21] J.B. Rosser, A. Turquette, Many-Valued Logics, North Holland Publ. Company, Amsterdam 1952

[22] G. Rousseau, Sequents in Many-valued logics I, Fundamenta Mathematica LX (1967), 23-33.

[23] K. Schröter, Methoden zur Axiomatisierung beliebiger Aussagen- und Prädikatenkalküle, Zeitschrift für math. Logik und Grundlagen der Mathematik 1 (1955), 241-251.

[24] R. Zach, Proof Theory of Finite-valued Logics, Master's thesis, Technische Universität Wien, 1993 (Available as Technical Report TUW-E185.2-Z.1-93).

Arnon Avron

School of Computer Science, Tel-Aviv University, Tel-Aviv, Israel

e-mail: aa@cs.tau.ac.il

Jonathan Ben-Naim

Centre de Mathématiques et Informatique de Marseille, France

e-mail: jbennaim@lif.univ-mrs.fr

Beata Konikowska

Institute of Computer Science, Polish Academy of Sciences, Warsaw, Poland

e-mail: beatak@ipipan.waw.pl 